IFUM-831-FT

\title{
Endowing the nonlinear sigma model with a flat connection structure: a way to renormalization
}

\author{
Ruggero Ferrari 1 \\ Physics Dept. University of Milan, \\ via Celoria 16, 20133 Milan, Italy \\ I.N.F.N., sezione di Milano
}

\begin{abstract}
We discuss the quantized theory of a pure-gauge non-abelian vector field (flat connection) as it would appear in a mass term à la Stückelberg. However the paper is limited to the case where only the flat connection is present (no field strength term). The perturbative solution is constructed by using only the functional equations and by expanding in the number of loops. In particular we do not use a perturbative approach based on the path integral or on a canonical quantization. It is shown that there is no solution with trivial S-matrix.

Then the model is embedded in a nonlinear sigma model. The solution is constructed by exploiting a natural hierarchy in the functional equations given by the number of insertions of the flat connection and of the constrained component of the sigma field. The amplitudes with the sigma field are simply derived from those of the flat connection and of the constraint component. Unitarity is enforced by hand by using Feynman rules. We demonstrate the remarkable fact that in generic dimensions the naïve Feynman rules yield amplitudes that satisfy the functional equations. This allows a dimensional renormalization of the theory in $\mathrm{D}=4$ by recursive subtractions of the poles in the Laurent expansion. Thus one gets a finite theory depending only on two parameters.

The novelty of the paper is the use of the functional equation associated to the local left multiplication introduced by Faddeev and Slavnov, here improved by adding the external source coupled to the constrained component. It gives a powerful tool to renormalize the nonlinear sigma model.
\end{abstract}

\footnotetext{
${ }^{1}$ E-mail address: ruggero.ferrari@mi.infn.it
} 


\section{Introduction}

Gauge field theories have been very successful in the description of the Physics of the sub-nuclear world. At the same time remarkable progresses have been achieved on their formulation, on the properties of the perturbative expansion, on the relevance of the invariance principles and on the importance of non-perturbative effects. In this effort for the construction of a correct theoretical foundation a central rôle is played by the requirement of Physical Unitarity [1]. The story goes back to the Gupta-Bleuler formulation of QED, where it is necessary to show that the unphysical modes do decouple from the physical states. The same strategy has been successful in the abelian gauge theory in presence of spontaneous breakdown of the symmetry (Higgs-Kibble model [2]), where the "gauge" mode of vector field conspires with the "phase" degree of freedom of the scalar field in order to restore Physical Unitarity. On the same road a solution has been found for the problem of Physical Unitarity in non-abelian gauge theories (also in presence of spontaneous breakdown). In this case one had to wait for clever solution based on the work of 't Hooft and Veltman 3. This progress has been possible after the introduction of the Faddeev-Popov ghosts [4]. The whole matter has been beautifully formulated after the discovery of the properties of Becchi-Rouet-Stora-Tyutin [5] symmetry. Also in this case the unphysical modes conspire in order to save Physical Unitarity.

Unphysical modes are introduced into the gauge theories in the process of quantization. Eventually they are described by pure-gauge vector fields.

While there are no secrets about the properties of the pure-gauge abelian field, the same cannot be said for non-abelian gauge. If one introduces a mass term à la Stückelberg [6], a pure-gauge field (flat connection) is needed that transforms according to

$$
F_{\mu}^{\prime}=U F_{\mu} U^{\dagger}+\frac{i}{g} U \partial_{\mu} U^{\dagger}
$$

and its field strength is zero

$$
\partial_{\mu} F_{\nu}-\partial_{\nu} F_{\mu}-i g\left[F_{\mu}, F_{\nu}\right]=0 .
$$

The group transformations are generated from the algebra

$$
\begin{aligned}
& {\left[t_{a}, t_{b}\right]=i f_{a b c} t_{c},} \\
& \operatorname{tr}\left(t_{a} t_{b}\right)=\kappa \delta_{a b}
\end{aligned}
$$

and

$$
F_{\mu}=t_{a} F_{a \mu} .
$$


In this work we will study some general properties of the flat connection. Then we will consider its relation with the nonlinear sigma model [7, 8, and discuss the difficulties connected with the presence of a non-trivial Haar measure and with the compact domain in the functional integration. We suggest to abandon the usual perturbation theory based on gaussian integrals and to use instead the functional identities associated to the transformations in eq. (11). We exemplify our procedure at the tree level (which agrees with a naïve approach). The one loop amplitudes are fixed in order to guarantee unitarity of the S-matrix [9, 10]. Amplitudes up to four-point are evaluated. We discuss also some two-loop amplitudes.

The key discovery is that the naïve D-dimension Feynman rules yield amplitudes that satisfy the functional equation. This fact is demonstrated in few cases. The importance of this fact is in the possibility of subtracting recursively the poles in the Laurent expansion, thus getting a finite theory. The amplitudes are given in terms of two parameters: coupling constant and mass.

The plan of the paper is the following. In Section 2 we briefly discuss the functional equations where only the flat connection is present. We argue that the flat connection cannot describe a scalar particle with trivial dynamics $(S \neq 1)$. In Section 3 the flat connection field is parametrized by a nonlinear sigma model. The notations are fixed and the Haar measure is discussed. In Section 4 we put in evidence the difficulties in the quantization of the theory by using perturbation theory in the path integral approach. Essentially one cannot avoid the problems generated by the presence of the Haar measure. In Section 5 we suggest a new strategy from start: use the functional equations in order to define the theory. In Section 6] we conjecture that the naïve Feynman rules yield a perturbative solutions of the functional equations in generic $\mathrm{D}$ dimensions. This allows a recursive subtraction of the poles in $D=4$, thus yielding a finite theory. This procedure of regularization is in the spirit of renormalization in the modern sense of [11]. In Section 7 we discuss the hierarchy of the functional equations, that can be ordered according to the number of flat connections and other composite operators. In Section 8 we discuss some standard aspects of the loop-expansion and in Section 9 we evaluate some tree-level amplitudes. In Section 10 we recall the standard procedure for the evaluation of connected amplitudes in terms of those 1PI. In Section 11 we consider few one-loop corrections and demonstrate the essential point of the discovery, i.e. that the perturbative expansion with standard Feynman rules in D dimensions yield amplitudes that satisfy the functional equations. In Section 12 we discuss the same problem for a two-loop two-point amplitude and Section 13 we outline the proof for the general case. In Section 14 we illustrate the subtraction procedure in few examples. 


\section{General properties}

Let us consider the path integral approach for the construction of the functional

$$
Z[J]=e^{i W[J]}=\int \mathcal{D}[F] \exp i \int d^{D} x\left(\frac{m_{D}^{2}}{8} F_{a \mu} F_{a}^{\mu}+F_{a \mu} J_{a}^{\mu}\right),
$$

where

$$
m_{D} \equiv m^{\frac{D-2}{2}}
$$

and the functional measure is supposed to be invariant under the transformations in eq. (11). The complexity of the problem is all hidden in the integration measure, since the action is a harmless quadratic form in the field. In general the integral cannot be performed analytically. Therefore we require only the validity of the functional identity associated to the transformation in eq. (1). For the generating functional of the connected amplitude $W$ we get 12

$$
\left(\frac{1}{4} m_{D}^{2} \partial^{\mu} W_{J_{a}^{\mu}}+\mathcal{D}_{a b \mu}\left[W_{J}\right] J_{b}^{\mu}\right)(x)=0,
$$

where

$$
\begin{aligned}
& \mathcal{D}_{a b \mu}[X] \equiv \partial_{\mu} \delta_{a b}-g f_{a b c} X_{c \mu} \\
& \mathcal{D}_{\mu}[X] \omega \equiv t_{a} \mathcal{D}_{a b \mu}[X] \omega_{b}=\partial_{\mu} \omega-i g\left[X_{\mu}, \omega\right] .
\end{aligned}
$$

We use the simplified notation

$$
\begin{aligned}
& W_{a_{1} \ldots a_{n}}^{\mu_{1} \ldots \mu_{n}}\left(x_{1}, \ldots, x_{n}\right)=\frac{\delta^{n} W}{\delta J_{a_{1} \mu_{1}}\left(x_{1}\right) \ldots \delta J_{a_{n} \mu_{n}}\left(x_{n}\right)} \\
& \int d^{D} x_{1} \ldots d^{D} x_{n} \exp i\left(p_{1} x_{1}+\ldots+p_{n} x_{n}\right) W_{a_{1} \ldots a_{n}}^{\mu_{1} \ldots \mu_{n}}\left(x_{1}, \ldots, x_{n}\right) \\
& =W_{a_{1} \ldots a_{n}}^{\mu_{1} \ldots \mu_{n}}\left(p_{1}, \ldots, p_{n}\right)(2 \pi)^{D} \delta\left(\sum_{j} p_{j}\right)
\end{aligned}
$$

The functional identity relates the connected amplitudes with different number of points. The relation for $n>2$ is ( "means "omitted")

$$
\begin{aligned}
& \frac{m_{D}^{2}}{4} p_{k \mu_{k}} W_{a_{1} \ldots a_{k} \ldots a_{n}}^{\mu_{1} \ldots \mu_{k} \ldots \mu_{n}}\left(p_{1}, \ldots, p_{k}, \ldots, p_{n}\right) \\
& =i g \sum_{j \neq k} f_{a_{k} a_{j} a_{j}^{\prime}} W_{a_{1} \ldots a_{j}^{\prime} \ldots \hat{a}_{k} \ldots a_{n}}^{\mu_{1} \ldots \mu_{j} \ldots \hat{\mu}_{k} \ldots \mu_{n}}\left(p_{1}, \ldots, p_{j}+p_{k}, \ldots, \hat{p}_{k}, \ldots, p_{n}\right)
\end{aligned}
$$

For $\mathrm{n}=2$ we have

$$
\frac{m_{D}^{2}}{4} p_{\mu} W_{a b}^{\mu \nu}(p)=-p^{\nu} \delta_{a b}
$$


There is a trivial solution of eqs. (11) and (12), i.e. all amplitudes are zero except

$$
W_{a b}^{\mu \nu}(p)=-\frac{4}{m_{D}^{2}} \delta_{a b} g^{\mu \nu} .
$$

This solution is formally given by the functional

$$
W[J]=-\int d^{D} x \frac{2}{m_{D}^{2}} J_{a}^{\mu} J_{a \mu}
$$

i.e.

$$
Z[J]=\int \mathcal{D}[F] \exp i \int d^{D} x\left(\frac{m_{D}^{2}}{8} F_{a \mu} F_{a}^{\mu}+J_{a \mu} F_{a}^{\mu}\right)
$$

where the integration is over the unconstrained configurations of the classical field $F_{a \mu}$.

The other possible solution is

$$
W_{a b}^{\mu \nu}(p)=\delta_{a b}\left(\left(g^{\mu \nu}-\frac{p^{\mu} p^{\nu}}{p^{2}}\right) W_{T}\left(p^{2}\right)-\frac{4}{m_{D}^{2}} \frac{p^{\mu} p^{\nu}}{p^{2}}\right)
$$

where the longitudinal part is exact. It would be tempting to construct a solution where $W_{T}\left(p^{2}\right)=0$ and to recursively deduce all other amplitudes from eq. (11) with the constraint that the S-matrix is equal one (no dynamics).

With these requirements one can easily construct the three point function. One gets

$$
\begin{aligned}
& W_{a_{1} a_{2} a_{3}}^{\mu_{1} \mu_{3}}\left(p_{1}, p_{2}, p_{3}\right)=-i g \frac{8}{m_{D}^{4}} f_{a_{1} a_{2} a_{3}}\left(\frac{p_{1}^{\mu_{1}} p_{2}^{\mu_{2}}}{p_{1}^{2} p_{2}^{2}}\left(p_{1}-p_{2}\right)^{\mu_{3}}\right. \\
& \left.+\frac{p_{3}^{\mu_{3}} p_{2}^{\mu_{2}}}{p_{3}^{2} p_{2}^{2}}\left(p_{2}-p_{3}\right)^{\mu_{1}}+\frac{p_{1}^{\mu_{1}} p_{3}^{\mu_{3}}}{p_{1}^{2} p_{3}^{2}}\left(p_{3}-p_{1}\right)^{\mu_{2}}\right) .
\end{aligned}
$$

However in evaluating the four point amplitude one finds an obstruction: there is no solution of eq. (8) under the assumption that $W_{T}\left(p^{2}\right)=0$, i.e. trivial S-matrix. Unfortunately the algebra is rather cumbersome and it isn't worth to elaborate the explicit calculation. The next section provides better technical instruments in order to investigate the whole problem of the construction of the connected amplitudes.

Since we cannot construct a trivial theory based on eq. (11) we have to take into account radiative corrections. Thus we consider a loop expansion where the transverse part in eq. (16) is zero at the tree level. With this assumption we evaluate the zero loop amplitudes. Then we proceed to evaluate the one-loop amplitudes by using the zero-loop results as Feynman rules (unitarity). The best tool for these calculations is provided by the 1PI amplitudes. 


\section{Flat connection and nonlinear sigma model}

The constraints in eqs. (10) and (2) can be implemented by using a field $\Omega(x)$ with value in a unitary group $G$ :

$$
F_{\mu}=\frac{i}{g} \Omega \partial_{\mu} \Omega^{\dagger}=t_{a} F_{a \mu}
$$

with

$$
\Omega^{-1}=\Omega^{\dagger}
$$

We parametrize the group with a set of real fields $\left\{\phi_{a}\right\}$. The action in (6) becomes

$$
\begin{aligned}
& S=\int d^{D} x \frac{m_{D}^{2}}{8} F_{a \mu} F_{a}^{\mu}=\int d^{D} x \frac{m_{D}^{2}}{8 g^{2} \kappa} \operatorname{tr}\left(\partial_{\mu} \Omega \partial^{\mu} \Omega^{\dagger}\right) \\
& =\int d^{D} x \frac{m_{D}^{2}}{8 g^{2} \kappa} \operatorname{tr}\left(\frac{\partial \Omega}{\partial \phi_{a}} \frac{\partial \Omega^{\dagger}}{\partial \phi_{b}}\right) \partial_{\mu} \phi_{a} \partial^{\mu} \phi_{b} \\
& =\int d^{D} x \frac{m_{D}^{2}}{2} \eta_{a b}(\phi) \partial_{\mu} \phi_{a} \partial^{\mu} \phi_{b}
\end{aligned}
$$

where

$$
\eta_{a b}=\frac{1}{4 g^{2} \kappa} \operatorname{tr}\left(\frac{\partial \Omega}{\partial \phi_{a}} \frac{\partial \Omega^{\dagger}}{\partial \phi_{b}}\right)
$$

with the property (as a consequence of unitarity)

$$
\eta_{a b}=\eta_{b a} .
$$

The nonlinear sigma model is of particular relevance in quantum field theory. It is the fundamental ingredient of some phenomenological models. It appears as a component or as a limit in theoretical models in field theory and in statistical field theory.

We consider the nonlinear sigma model for $S U(2)$ in order to keep the notations as simple as possible. Most of the results can be generalized to other groups. We introduce the parametrization

$$
\Omega=\frac{1}{m_{D}}\left[\phi_{0}+i g \tau_{a} \phi_{a}\right] \quad t_{a}=\frac{\tau_{a}}{2} \quad a=1,2,3 .
$$

Eq. (19) and

$$
\operatorname{det} \Omega=1
$$

gives

$$
\phi_{0}^{2}+g^{2} \vec{\phi}^{2}=m_{D}^{2}
$$


Then

$$
\begin{gathered}
\eta_{a b}=m_{D}^{-2}\left(g^{2} \frac{\phi_{a} \phi_{b}}{\phi_{0}^{2}}+\delta_{a b}\right), \\
S=\frac{1}{2} \int d^{D} x\left(\partial^{\mu} \phi_{a} \partial_{\mu} \phi_{a}+g^{2} \frac{\phi_{a} \partial^{\mu} \phi_{a} \phi_{b} \partial_{\mu} \phi_{b}}{\phi_{0}^{2}}\right) \\
=\frac{1}{2} \int d^{D} x\left(\partial^{\mu} \phi_{a} \partial_{\mu} \phi_{a}+\frac{1}{g^{2}} \partial^{\mu} \phi_{0} \partial_{\mu} \phi_{0}\right)
\end{gathered}
$$

and

$$
\eta \equiv \operatorname{det} \eta=\frac{1}{m_{D}^{4} \phi_{0}^{2}}
$$

The constraint (25) and the action (27) are invariant under the global transformations

$$
\begin{aligned}
\delta \phi_{0} & =-g^{2} \frac{\delta \omega_{a}}{2} \phi_{a} \\
\delta \phi_{a} & =\frac{\delta \omega_{a}}{2} \phi_{0}+g \frac{\delta \omega_{c}}{2} \epsilon_{a b c} \phi_{b} .
\end{aligned}
$$

Both constraint and action are also invariant under the global transformations

$$
\begin{aligned}
\delta \phi_{0} & =0 \\
\delta \phi_{a} & =\frac{\delta \alpha_{c}}{2} \epsilon_{a b c} \phi_{b} .
\end{aligned}
$$

The transformations in eqs. (29) and (30) describe the invariance of the model under $S U(2) \otimes S U(2)$ group of transformations.

The transformations in eq. (29) are given by the left multiplication

$$
\Omega^{\prime}=U(\delta \omega) \Omega \simeq\left(1+i \frac{g}{2} \tau_{a} \delta \omega_{a}\right) \Omega .
$$

The flat connection is

$$
F_{a \mu}=\frac{i}{g} \operatorname{tr}\left(\tau_{a} \Omega \partial_{\mu} \Omega^{\dagger}\right)=\frac{2}{m_{D}^{2}}\left[\left(\phi_{0} \partial_{\mu} \phi_{a}-\left(\partial_{\mu} \phi_{0}\right) \phi_{a}\right)+g \epsilon_{a b c}\left(\partial_{\mu} \phi_{b}\right) \phi_{c}\right] .
$$

The properties of the flat connection under local transformations (29) is that of a gauge field (1)

$$
\begin{aligned}
F_{\mu}^{\prime} & =U F_{\mu} U^{\dagger}+\frac{i}{g} U \partial_{\mu} U^{\dagger} \\
& \simeq F_{\mu}+i g\left[\omega, F_{\mu}\right]+\partial_{\mu} \omega, \quad \text { with } \omega=\frac{\tau_{a}}{2} \omega_{a} \\
& =F_{\mu}+\mathcal{D}_{\mu}[F] \omega
\end{aligned}
$$


where the covariant derivative is defined in (9).

The transformations given by the right multiplication

$$
\Omega^{\prime}=\Omega U^{\dagger}(\delta \bar{\omega}) \simeq \Omega\left(1-i \frac{g}{2} \tau_{a} \delta \bar{\omega}_{a}\right)
$$

yield

$$
\begin{aligned}
& \delta \phi_{0}=g^{2} \frac{\delta \bar{\omega}_{a}}{2} \phi_{a} \\
& \delta \phi_{a}=-\frac{\delta \bar{\omega}_{a}}{2} \phi_{0}+g \frac{\delta \bar{\omega}_{c}}{2} \epsilon_{a b c} \phi_{b} .
\end{aligned}
$$

For "local" right multiplication the flat connection transforms into new composite operators, therefore they produce functional identities which are not useful for the construction of the generating functionals.

The paper concerns the construction of the n-point function of the flat connection

$$
\left\langle 0\left|T\left(F_{a_{1}}^{\mu_{1}}\left(x_{1}\right) \cdots F_{a_{n}}^{\mu_{n}}\left(x_{n}\right)\right)\right| 0\right\rangle .
$$

The construction of these n-functions will require the study of the functions

$$
\left\langle 0\left|T\left(\phi_{a_{1}}\left(x_{1}\right) \cdots \phi_{a_{n}}\left(x_{n}\right)\right)\right| 0\right\rangle .
$$

Thus we introduce the external sources by the term

$$
S_{\mathrm{sc}}=\int d^{D} x\left(\phi_{0} K_{0}+\phi_{a} K_{a}+F_{a \mu} J_{a}^{\mu}\right) .
$$

In the paper we discuss the construction of the solution of eq. (8) by considering the loop expansion of the connected amplitudes and of the vertex functions (1PI amplitudes). The iteration procedure is chosen in order to satisfy unitarity of the scattering amplitude. The necessity of this procedure is due to the complicated (non-polynomial) structure of the action and on the fact that the path integral approach has a non-trivial Haar measure in the functional integration. The next sections are devoted to clarify the above points.

\section{In straits}

The importance of the nonlinear sigma model is hindered by the difficulties present in the procedure of quantization. The difficulties come mainly from the constraint on the field components, under various aspects. Let us elaborate on this point, since it is the starting point of our approach. The path integral formulation of the quantized theory starts from a formal definition of the generating functional. 
The path integral approach to the quantization of the theory needs the introduction of an invariant measure over the group

$$
Z\left[K, K_{0}, J\right]=\int \mathcal{D}[\Omega] \exp i\left(S+S_{\mathrm{sc}}\right) .
$$

In terms of parameter fields we have

$$
Z\left[K, K_{0}, J\right]=\int \prod_{x} \sqrt{\operatorname{det} \eta(\phi)} d \phi(x) \exp i\left(S+S_{\mathrm{sc}}\right) .
$$

For instance the measure in the case of equation (28) is given by

$$
Z\left[K, K_{0}, J\right]=\prod_{x} \int_{g^{2}|\phi|^{2}<m_{D}^{2}} \frac{d^{3} \phi(x)}{\phi_{0}} \exp i\left(S+S_{\mathrm{sc}}\right) .
$$

The path integral in eq. (41) shows some peculiarities: a non-trivial Haar measure is present and the integration over the field is on a compact region. These facts cause some difficulties in formulating a perturbative expansion by using the path integral. We would like to illustrate these difficulties. If we use a straightforward series expansion of the exponential containing the "interaction", the quantities to be evaluated are

$$
\prod_{x} \int_{g^{2}|\phi|^{2}<m_{D}^{2}} \frac{d^{3} \phi(x)}{\phi_{0}}\left(\phi_{a_{1}}\left(y_{1}\right) \ldots \phi_{a_{m}}\left(y_{m}\right)\right) \exp \left(\frac{i}{2} \int d^{D} z \partial_{\mu} \phi_{a} \partial^{\mu} \phi_{a}\right) .
$$

Here the integrals are not Gaussian and therefore the Wick expansion is in general not valid. The evaluation of the path integrals over a group manyfold are in general beyond the present day technical ability, for anything beyond the two-point function 13 .

In alternative to the above expansion, it has been suggested that one should expand in the coupling constant $g$. Therefore the Haar measure should be treated by the exponentiation and moreover that the integration can be extended to infinity. A careful analysis of the divergences in the path integral formulation has shown a remarkable cancellation between the divergent terms coming from the Haar measure and some of the divergences of the action [14]. In particular the most severe divergent terms are reabsorbed by a redefinition of the Weinberg's function $f\left(\vec{\phi}^{2}\right)[15]$. This indicates that the principal rôle of the action in the renormalization process has to be somehow supplemented or enlarged to include a redefinition of the fields.

A clear cut solution of the problem has been proposed after the discovery of dimensional regularization [16]. In studying the renormalization of the nonlinear sigma model in two dimension Brezin Zinn-Justin le Guillou [17] discussed the problem of the non trivial Haar measure and suggested that dimensional renormalization provides a solution. On the same line 't Hooft [18] noticed that all the infinities coming from the Haar measure could be 
disposed of by dimensional renormalization. The approach based on the exponentiation of the Haar measure deals with ill defined objects (usually $\delta(0))$ and moreover the integration over the fields is taken on the whole real axis by considering the approximation of small $g$ for the limit of integration $\sim 1 / g$. The final output of these approximations is untenable, since the generating functional yields an equation of motion that is not in agreement with the exact functional equation derived from the local invariance of the Haar measure, as discussed in the next Section (see in particular the footnote on the eq. (45)).

A further serious difficulty in the quantization of the nonlinear sigma model was pointed out by Tătaru [19]. In the one-loop amplitude in dimensional renormalization terms show up that violate manifestly the global invariance. Appelquist and Bernard [20] showed that these terms can be reabsorbed by reparametrization of the scalar field. This reparametrization however includes space-time derivatives i.e. is not of the form in eq. (201).

To the best of our knowledge this was the last contribution to the problem of the quantization of the nonlinear sigma model. However the model has been considered in many different phenomenological schemes, where the problem of regularization has been addressed. We mention the chiral field theory [21] where the Matching Conditions method [22] has been used. In the next section we suggest an alternative approach to the construction of perturbative solution of the nonlinear sigma model by using the n-point flat connection amplitudes.

\section{Deliverance}

In the previous section we have pointed out the difficulty in dealing with perturbation theory if a non-trivial Haar measure is present in the path integral and the integration over the fields is on a compact support.

We try to pursue a different approach. By starting from the fact that our goal is the evaluation of the correlation functions, we might try to get them by solving directly the functional equations as an alternative to formal integration and subsequent perturbative expansion in terms of Gaussian integrals.

Since the functional given by functional integration in eq. (39) is a sum over the group of transformations $\Omega$ we conclude that the extremal should be found inside the set of configurations $\{\Omega(x)\}$. Therefore the infinitesimal variation of the field variables should be constrained to the set given by eq. (25). In general we consider the variation (29)

$$
\delta \Omega(x)=i g \delta \omega_{a}(x) t_{a} \Omega(x) .
$$

The identity for the generating functional $Z$ (or for that of the connected 


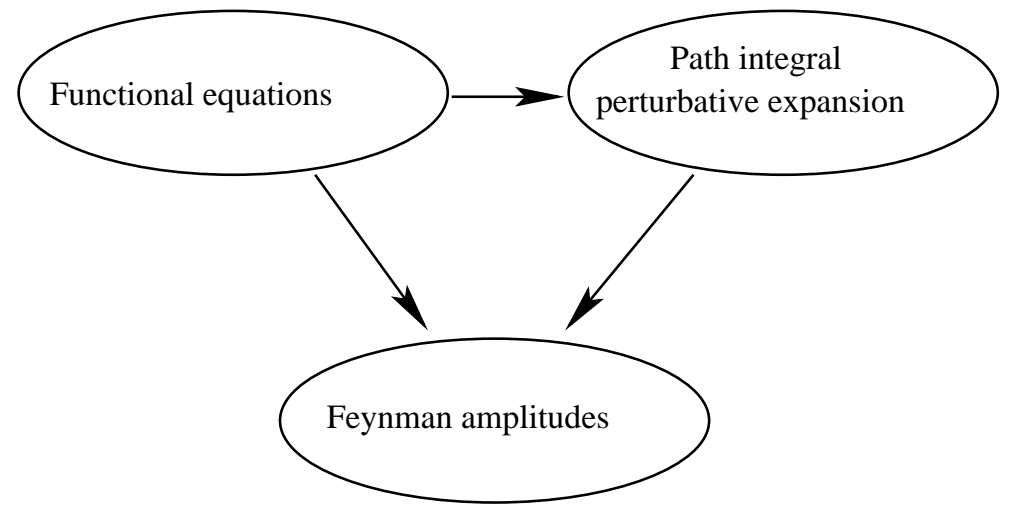

Figure 1: Alternatives in the construction of Feynman amplitudes

$W)$ is 2

$$
\begin{aligned}
& \int \mathcal{D}[\Omega] \exp \left(i\left(S+S_{\mathrm{sc}}\right)\right)\left(\frac{i}{4 g \kappa} \operatorname{tr}\left(-t_{a} \Omega \square \Omega^{\dagger}+\square \Omega \Omega^{\dagger} t_{a}\right)\right. \\
& \left.-g^{2} \phi_{a} K_{0}+\phi_{0} K_{a}+g \epsilon_{a b c} K_{b} \phi_{c}+2 \mathcal{D}_{a b \mu}[F] J_{b}^{\mu}\right)(x) \\
& =\int \mathcal{D}[\Omega] \exp \left(i\left(S+S_{\mathrm{sc}}\right)\right)\left(\left(\phi_{a} \square \phi_{0}-\square \phi_{a} \phi_{0}\right)+g \epsilon_{a b c} \phi_{b} \square \phi_{c}\right. \\
& \left.-g^{2} \phi_{a} K_{0}+\phi_{0} K_{a}+g \epsilon_{a b c} K_{b} \phi_{c}-2 \mathcal{D}_{a b \mu}[F] J_{b}^{\mu}\right)(x)=0 .
\end{aligned}
$$

From eq. (32) we get

$$
\begin{aligned}
& \int \mathcal{D}[\Omega] \exp \left(i\left(S+S_{\mathrm{sc}}\right)\right)\left(-\frac{m_{D}^{2}}{2} \partial^{\mu} F_{a \mu}\right. \\
& \left.-g^{2} \phi_{a} K_{0}+\phi_{0} K_{a}+g \epsilon_{a b c} K_{b} \phi_{c}-2 \mathcal{D}_{a b \mu}[F] J_{b}^{\mu}\right)(x)=0 .
\end{aligned}
$$

Thus the identity for the generating functional of the connected amplitudes is (in $D$ dimensions)

$$
\begin{aligned}
& \left(-\frac{m_{D}^{2}}{2} \partial^{\mu} \frac{\delta W}{\delta J_{a}^{\mu}}-g^{2} \frac{\delta W}{\delta K_{a}} K_{0}+\frac{\delta W}{\delta K_{0}} K_{a}+g \epsilon_{a b c} K_{b} \frac{\delta W}{\delta K_{c}}\right. \\
& \left.-2 \mathcal{D}_{a b \mu}\left[\frac{\delta W}{\delta J}\right] J_{b}^{\mu}\right)(x)=0 .
\end{aligned}
$$

${ }^{2}$ Notice the difference with the usual equations of motion obtained by a change of coordinates $\phi_{a} \rightarrow \phi_{a}+\delta \phi_{a}$ (for semplicity we take $J_{a \mu}=0$ )

$$
\int \mathcal{D}[\vec{\phi}] \exp i\left(S+\int d^{D} z K_{0}(z) \phi_{0}(z)\right)\left(\left(\frac{1}{\phi_{0}} \square \phi_{0} \phi_{a}-\square \phi_{a}\right)+K_{a}-g^{2} \frac{1}{\phi_{0}} K_{0} \phi_{a}\right)(x)=0,
$$

which requires a set of new composite operator sources. 
For the generating functional of the 1PI amplitudes one has

$$
\begin{aligned}
& \frac{\delta \Gamma\left[J, K_{0}, \vec{\phi}\right]}{\delta J_{a}^{\mu}}=\frac{\delta W\left[J, K_{0}, \vec{K}\right]}{\delta J_{a}^{\mu}} \\
& \frac{\delta \Gamma\left[J, K_{0}, \vec{\phi}\right]}{\delta K_{0}}=\frac{\delta W\left[J, K_{0}, \vec{K}\right]}{\delta K_{0}}
\end{aligned}
$$

and therefore

$$
\begin{aligned}
& \left(\frac{m_{D}^{2}}{2} \partial^{\mu} \frac{\delta \Gamma}{\delta J_{a}^{\mu}}+g^{2} \phi_{a} K_{0}+\frac{\delta \Gamma}{\delta K_{0}} \frac{\delta \Gamma}{\delta \phi_{a}}+g \epsilon_{a b c} \frac{\delta \Gamma}{\delta \phi_{b}} \phi_{c}\right. \\
& \left.+2 \mathcal{D}\left[\frac{\delta \Gamma}{\delta J}\right]_{a b}^{\mu} J_{b \mu}\right)(x)=0 .
\end{aligned}
$$

By using eqs. (47) and (49) we shall try to find the n-point function of the flat connection $F_{\mu}$. Since we do not solve these equations with the help of functional integration, unitarity has to be enforced at every order in the loop expansion. This will be achieved by performing contractions on the external legs with the Feynman prescription on the complex integration for the propagators.

Eqs. (47) and (49) establish an important hierarchy in the construction of the perturbative solution. Once the amplitudes for flat connections and for $\phi_{0}$ are evaluated, those involving the field $\vec{\phi}$ are derived by using eqs. (47) and (49) in a descending order: at each step one gets one more $\vec{\phi}$ field and one less flat connection. This somehow takes into account automatically the arbitrariness in the parametrization of the field $\Omega$ in terms of $\vec{\phi}$. Different parameterizations yield different functional equations, i.e. different Feynman rules involving the nonlinear sigma model.

The renormalization via analytic continuation in the space-time dimensions must take into account that eqs. (47) and (49) carry a dimensional parameter $m$. With a simple dimensional argument one sees that the minimal subtraction (poles in $D=4$ ) has to be done on the normalized amplitudes

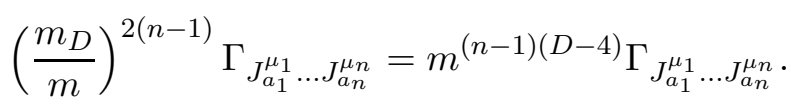

For the amplitudes involving the fields $\vec{\phi}$ and $\phi_{0}$ eqs. (47) and (49) provide the correct $m_{D}$ factor.

\section{Renormalization}

In this section we present some personal view on renormalization. We follow very close the idea of "renormalization in the modern sense" of Reference [11. We consider of particular interest the theories that can be made finite in a symmetric fashion, i.e. by preserving the salient properties as unitarity, 
Lorentz covariance, locality, causality and in general equations of motion and symmetries under the form of functional identities for the generating functionals e.g. Ward identities, Slavnov-Taylor identities, etc.. This is a pure mathematical problem which might have many solutions. From the physical point of view a particular solution can be proposed as a dynamical model on many different grounds, even that of elegance. Any solution is in general a predictive model if it is supposed to describe physical modes. However there are also other interesting possibilities as for instance the case of theories where the multiplicity of the solutions affects only the unphysical modes.

A subset of these renormalizable theories have a unique perturbative solution once a finite number of parameters are fixed (i.e. those that one indicates usually as "renormalizable"). In the present work we show that the nonlinear sigma model can be renormalized in the wider meaning just introduced.

The amplitudes are expanded in the number of loops (or equivalently in powers of the coupling constant $g$ ). The lowest terms of this expansions are given by the tree graphs and can be obtained directly by solving eq. (47) or (49). This is exemplified in Section 9. There is an alternative approach to the construction of the tree level amplitudes, more close to our renormalization strategy, as we outline in this section. By straightforward calculation it can be proved that

$$
\begin{aligned}
& \left(\frac{m_{D}^{2}}{2} \partial^{\mu} \frac{\delta \Gamma^{(0)}}{\delta J_{a}^{\mu}}+g^{2} \phi_{a} K_{0}+\frac{\delta \Gamma^{(0)}}{\delta K_{0}} \frac{\delta \Gamma^{(0)}}{\delta \phi_{a}}+g \epsilon_{a b c} \frac{\delta \Gamma^{(0)}}{\delta \phi_{b}} \phi_{c}\right. \\
& \left.+2 \mathcal{D}\left[\frac{\delta \Gamma^{(0)}}{\delta J}\right]_{a b}^{\mu} J_{b \mu}\right)(x)=0,
\end{aligned}
$$

where the classical vertex functional is

$$
\Gamma^{(0)}=\int d^{D} x\left\{\frac{1}{2}\left(\partial^{\mu} \phi_{a} \partial_{\mu} \phi_{a}+\frac{1}{g^{2}} \partial^{\mu} \phi_{0} \partial_{\mu} \phi_{0}\right)+K_{0} \phi_{0}+J_{a}^{\mu} F_{a \mu}\right\} .
$$

This result is very important since it saves a lot of work in the explicit evaluation of the tree level amplitudes for an arbitrary number of external legs. It is important to notice that for $J_{a}^{\mu}=0$ the Feynman rules derived from $\Gamma^{(0)}$ are symmetric under the transformation

$$
\phi_{a} \rightarrow-\phi_{a}
$$

The higher loop amplitudes are constructed by using the Feynman rules given by $\Gamma^{(0)}$ in eq.(52). We shall demonstrate that this approach yields a solution of eq. (49) (or (47)), provided one neglects all integrals representing a loop where no outside momentum flows as shown in Refs. [23]. I.e.

$$
\int d^{D} k \frac{1}{\left(k^{2}\right)^{\alpha}}=0, \quad \alpha \in \mathcal{C} .
$$


We suggest that the amplitudes in $D=4$ dimensions are obtained by iterative subtraction of the pole parts. By subtracting the pole parts of the subgraphs according to the BPHZ prescription one obtains a finite amplitude for any n-1 loop amplitude. The pole part at one loop level is expected to satisfy the linearized functional equation

$$
\begin{aligned}
& \left(\frac{m_{D}^{2}}{2} \partial^{\mu} \frac{\delta}{\delta J_{a}^{\mu}}+\frac{\delta \Gamma^{(0)}}{\delta \phi_{a}} \frac{\delta}{\delta K_{0}}+\frac{\delta \Gamma^{(0)}}{\delta K_{0}} \frac{\delta}{\delta \phi_{a}}+g \epsilon_{a b c} \phi_{c} \frac{\delta}{\delta \phi_{b}}\right. \\
& \left.-2 g \epsilon_{a b c} J_{b \mu} \frac{\delta}{\delta J_{c}^{\mu}}\right) \Gamma_{\mathrm{POLE}}^{(1)}=0 .
\end{aligned}
$$

At higher order the counterterms $\hat{\Gamma}^{(n)}$ are fixed according to hierarchy of eq. (49).

We summarize the renormalization strategy by the two-step procedure:

1. Enforce the validity of eqs. (47) and (49) in generic $D$ dimensions. This is achieved by neglecting all tadpole contributions as required by the rule in eq. (54). This point is crucial in our approach. Up to two loop the eq. (54) guarantees that the functional equations are satisfied by the naïve Feynman rules. For higher number of loops the situation is more complex and it is illustrated in Section [13, where it is shown in a specific example that the equation of motion given by the tree level of eq. (47)

$$
\begin{aligned}
& \left(-\frac{m_{D}^{2}}{2} \partial^{\mu} \frac{\delta W^{(0)}}{\delta J_{a}^{\mu}}-g^{2} \frac{\delta W^{(0)}}{\delta K_{a}} K_{0}+\frac{\delta W^{(0)}}{\delta K_{0}} K_{a}+g \epsilon_{a b c} K_{b} \frac{\delta W^{(0)}}{\delta K_{c}}\right. \\
& \left.-2 \mathcal{D}_{a b \mu}\left[\frac{\delta W^{(0)}}{\delta J}\right] J_{b}^{\mu}\right)(x)=0 .
\end{aligned}
$$

restores the validity of eq. (49) in D-dimensions.

2. Subtract recursively, as in BPHZ [24] renormalization procedure, the pole parts in the Laurent expansion in $D-4$ by using the local solutions of eq. (55) at the one loop level. For higher order the subtraction procedure requires a faithful acceptance of hierarchy. First fix the counterterms necessary for the subtraction of the poles in $D=4$ of the amplitudes involving only flat connection $\left(F_{a}^{\mu}\right)$ and $\phi_{0}$ (derivatives with respect to $K_{0}$ ) insertions normalized according to eq. (50). Then derive the counterterms for amplitudes involving also $\vec{\phi}$.

In Section 14 we provide some simple examples of this strategy. It should be stressed once more that the key point is that the functional equations (47) and (49) are valid in $D$ dimensions and therefore a symmetric subtraction is possible. This statement is valid for theories that are power counting renormalizable, where the constraint on the dimension of the counterterms plays a crucial rôle. Here we can only conjecture that the removal of the 
poles at $D=4$ does not conflict with the functional eqs. (47) and (49). In the present paper we verify the validity of the conjecture on a two-loop example $\left(\Gamma_{J J}^{(2)}, \Gamma_{J \phi}^{(2)}, \Gamma_{\phi \phi}^{(2)}\right)$.

We want to highlight once again few points about this strategy.

- Since $\Gamma^{(0)}$ is the 1PI functional then at the tree level the maximum number of $K_{0}$ or $J_{\mu}^{a}$ insertions is one as can be seen from eq. (52).

- For any radiative corrections the pole subtraction at the one loop level corresponds to a local solution of eq. (55). The same equation controls all the possible choices of dimensional renormalization. Therefore the study of the equation (55) is very important for the renormalization of the non linear sigma model. By defining

$$
\begin{aligned}
& \mathcal{S} \equiv \frac{m_{D}^{2}}{2} \partial^{\mu} \frac{\delta}{\delta J_{a}^{\mu}}+\frac{\delta \Gamma^{(0)}}{\delta \phi_{a}} \frac{\delta}{\delta K_{0}}+\frac{\delta \Gamma^{(0)}}{\delta K_{0}} \frac{\delta}{\delta \phi_{a}}+g \epsilon_{a b c} \phi_{c} \frac{\delta}{\delta \phi_{b}} \\
& -2 g \epsilon_{a b c} J_{b \mu} \frac{\delta}{\delta J_{c}^{\mu}}
\end{aligned}
$$

different choices of the local counterterms part $\widehat{\Gamma}, \widehat{\Gamma}^{\prime}$ have to satisfy the equation

$$
\mathcal{S}\left(\widehat{\Gamma}-\widehat{\Gamma}^{\prime}\right)=0
$$

- After the subtractions have been performed the finite amplitudes are given in terms of two parameters only: $g$ and $m$. Thus in principle the theory becomes predictive. However the dependence of the amplitudes from the parameters $g$ and $m$ is entangled with the choice of the finite parts that satisfy eq. (58).

\section{Hierarchy of the functional equations}

We will consider some n-point functions, that will be constructed by using the functional equations. The solution of eqs. (47) and (49) is given in terms of some parameters. The invariance properties of the equations allows to fix some of these at convenient values. For instance the invariance under

$$
\begin{aligned}
& K_{0} \rightarrow v K_{0} \\
& \vec{\phi} \rightarrow \frac{1}{v} \vec{\phi}
\end{aligned}
$$

allows to fix the condition

$$
\left.W_{K_{0}}\right|_{\vec{K}=K_{0}=J_{\mu}=0}=\left.\Gamma_{K_{0}}\right|_{\vec{K}=K_{0}=J_{\mu}=0}=m_{D}
$$


One can also consider the transformation

$$
\begin{aligned}
& J_{a \mu} \rightarrow g J_{a \mu} \\
& \vec{\phi} \rightarrow \frac{1}{g} \vec{\phi} \\
& m_{D} \rightarrow g m_{D}
\end{aligned}
$$

which removes the constant $g$ in eq. (49). However this transformation induces a $g$-dependence in eq. (60), which we prefer to avoid since eq. (60) fixes the spontaneous breakdown of the global symmetry under left multiplication expressed by eq. (29).

We shall consider the explicit functional derivatives of eq. (49). This will show the hierarchy implicit in the functional equation: once the function for the flat-connection and of the constrained component $\phi_{0}$ only is evaluated, the sigma model follows simply by successive derivatives. We list some of them where use is made of the condition (60).

\subsection{Two-point functions}

By taking one derivative of eq. (49)

$$
m_{D} \partial^{\mu} \Gamma_{J_{a}^{\mu} \phi_{b}}+2 \Gamma_{\phi_{a} \phi_{b}}=0
$$

and

$$
m_{D}^{2} \partial^{\mu} \Gamma_{J_{a}^{\mu} J_{b}^{\nu}}+2 m_{D} \Gamma_{\phi_{a} J_{b}^{\nu}}+4 \partial_{\nu} \delta(x-y) \delta_{a b}=0 .
$$

\subsection{Three-point functions}

By taking two derivatives we get

$$
\begin{aligned}
& \frac{m_{D}^{2}}{2} \partial^{\mu_{1}} \Gamma_{J_{a_{1}}^{\mu_{1}} \phi_{a_{2}} \phi_{a_{3}}}+m_{D} \Gamma_{\phi_{a_{1}} \phi_{a_{2}} \phi_{a_{3}}} \\
& +g\left(\epsilon_{a_{1} b a_{3}} \Gamma_{\phi_{b} \phi_{a_{2}}} \delta\left(x_{1}-x_{3}\right)-\epsilon_{a_{1} a_{2} b} \Gamma_{\phi_{b} \phi_{a_{3}}} \delta\left(x_{1}-x_{2}\right)\right)=0 . \\
& \frac{m_{D}^{2}}{2} \partial^{\mu_{1}} \Gamma_{J_{1} J_{a_{2}} J_{a_{3}}^{\mu_{2}}}+m_{D} \Gamma_{\phi_{a_{1}} J_{a_{2}}^{\mu_{2}} \phi_{a_{3}}}
\end{aligned}
$$

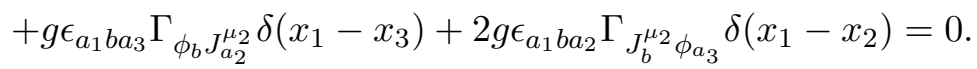

$$
\begin{aligned}
& \frac{m_{D}^{2}}{2} \partial^{\mu_{1}} \Gamma_{a_{1}}^{\mu_{1}} J_{a_{2}}^{\mu_{2}} J_{a_{3}}^{\mu_{3}}+m_{D} \Gamma_{\phi_{a_{1}}} J_{a_{2}}^{\mu_{2}} J_{a_{3}}^{\mu_{3}}
\end{aligned}
$$

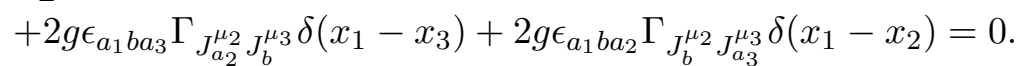


Similarly

$$
\begin{aligned}
& \frac{m_{D}^{2}}{2} \partial^{\mu_{1}} \Gamma_{J_{a_{1}}^{\mu_{1}} \phi_{a_{2}} K_{0}}+g^{2} \delta_{a_{1} a_{2}} \delta\left(x_{1}-x_{2}\right) \delta\left(x_{1}-x_{3}\right)+\Gamma_{K_{0} K_{0}} \Gamma_{\phi_{a_{1}} \phi_{a_{2}}} \\
& +m_{D} \Gamma_{\phi_{a_{1}} \phi_{a_{2}} K_{0}}=0
\end{aligned}
$$

and

$$
\frac{m_{D}^{2}}{2} \partial^{\mu_{1}} \Gamma_{J_{a_{1}}^{\mu_{1}} J_{a_{2}}^{\mu_{2}} K_{0}}+m_{D} \Gamma_{J_{a_{1}}^{\mu_{1}} \phi_{a_{2}} K_{0}}+\Gamma_{K_{0} K_{0}} \Gamma_{\phi_{a_{1}} J_{a_{2}}^{\mu_{2}}}=0
$$

\subsection{Four-point functions}

By taking three derivatives we get

$$
\begin{aligned}
& \frac{m_{D}^{2}}{2} \partial^{\mu_{1}} \Gamma_{J_{a_{1}}^{\mu_{1}} \phi_{a_{2}} \phi_{a_{3}} \phi_{a_{4}}}+m_{D} \Gamma_{\phi_{a_{1}} \phi_{a_{2}} \phi_{a_{3}} \phi_{a_{4}}} \\
& +\sum_{j=2}^{4} \Gamma_{K_{0} \phi_{a_{j+1}} \phi_{a_{j+2}}} \Gamma_{\phi_{a_{1}} \phi_{a_{j}}}+\sum_{j=2}^{4} g \epsilon_{a_{1} b a_{j}} \Gamma_{\phi_{b} \phi_{a_{j+1}} \phi_{a_{j+2}}} \delta\left(x_{1}-x_{j}\right)=0 .
\end{aligned}
$$

$$
\begin{aligned}
& \frac{m_{D}^{2}}{2} \partial^{\mu_{1}} \Gamma_{J_{a_{1}}^{\mu_{1}} J_{a_{2}}^{\mu_{2} \phi_{a_{3}} \phi_{a_{4}}}}+m_{D} \Gamma_{\phi_{a_{1}} J_{a_{2}}^{\mu_{2}} \phi_{a_{3}} \phi_{a_{4}}} \\
& +\Gamma_{K_{0} \phi_{a_{3}} \phi_{a_{4}}} \Gamma_{\phi_{a_{1}} J_{a_{2}}^{\mu_{2}}}+\sum_{j=3,4}\left(\Gamma_{K_{0} J_{a_{2}}^{\mu_{2}} \phi_{a_{j}}} \Gamma_{\phi_{a_{1}} \phi_{a_{(j+1)}}}\right. \\
& \left.+g \epsilon_{a_{1} b a_{j}} \Gamma_{\phi_{b} J_{a_{2}}^{\mu_{2}} \phi_{a_{(j+1)}}} \delta\left(x_{1}-x_{j}\right)\right)+2 g \epsilon_{a_{1} b a_{2}} \Gamma_{J_{b}^{\mu_{2}} \phi_{a_{3}} \phi_{a_{4}}} \delta\left(x_{1}-x_{2}\right)=0 .
\end{aligned}
$$

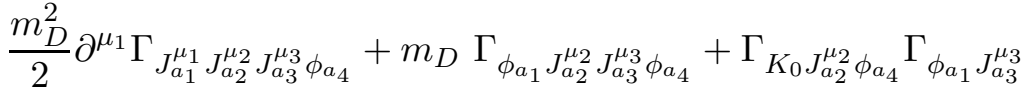

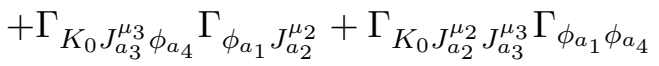

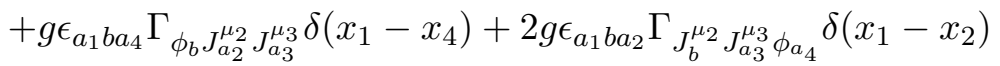

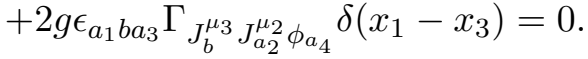

Finally we take only derivatives respect to $J_{a \mu}$

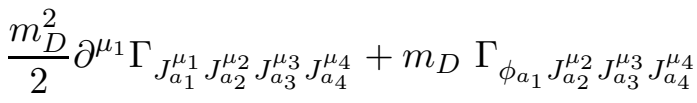

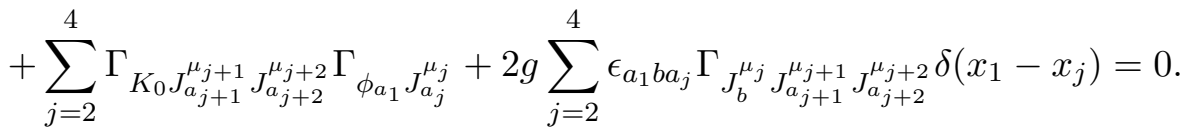




\section{Perturbative solution}

In Section 2 we have considered some properties of the amplitudes for a flat connection. In particular we have discovered that the longitudinal part of the two-point function gets no corrections from the higher terms in the loop expansion

$$
W_{J_{a}^{\mu} J_{b}^{\nu}}(p)=\delta_{a b}\left(\left(p^{2} g_{\mu \nu}-p_{\mu} p_{\nu}\right) W_{\mathrm{T}}\left(p^{2}\right)-\frac{4}{m_{D}^{2}} \frac{p_{\mu} p_{\nu}}{p^{2}}\right) .
$$

We expect the transverse part to be non zero at higher loop corrections. It is interesting to see how eq. (73) can be valid even in a theory with non-trivial dynamics. To see this we consider 1PI amplitudes. That is we use eqs. (47) and (49). For the generating functional of the connected amplitudes we have

$$
-\frac{m_{D}^{2}}{2} \partial^{\mu} W_{J_{a}^{\mu}(x) K_{b}(y)}+\delta_{a b} \delta(x-y) W_{K_{0}}=0
$$

i.e.

$$
W_{J_{a}^{\mu} K_{b}}=2 i \frac{p_{\mu}}{m_{D} p^{2}} \delta_{a b}
$$

For the 1PI amplitudes we get the following two-point functions

$$
m_{D} \partial^{\mu} \Gamma_{J_{a}^{\mu} \phi_{b}}+2 \Gamma_{\phi_{a} \phi_{b}}=0
$$

and

$$
m_{D}^{2} \partial^{\mu} \Gamma_{J_{a}^{\mu} J_{b}^{\nu}}+2 m_{D} \Gamma_{\phi_{a} J_{b}^{\nu}}+4 \partial_{\nu} \delta(x-y) \delta_{a b}=0 .
$$

We can now reconstruct the connected amplitude in terms of the 1PI ones.

$$
W\left[J, K_{0}, \vec{K}\right]_{J_{a}^{\mu} J_{b}^{\nu}}=\Gamma\left[J, K_{0}, \vec{\phi}\right]_{J_{a}^{\mu} J_{b}^{\nu}}+W\left[J, K_{0}, \vec{K}\right]_{J_{a}^{\mu} K_{c}} \Gamma\left[J, K_{0}, \vec{\phi}\right]_{\phi_{c} J_{b}^{\nu}}
$$

The longitudinal part satisfies the equation (12):

$$
\begin{aligned}
& \partial^{\mu} W\left[J, K_{0}, \vec{K}\right]_{J_{a}^{\mu} J_{b}^{\nu}}=\partial^{\mu} \Gamma\left[J, K_{0}, \vec{\phi}\right]_{J_{a}^{\mu} J_{b}^{\nu}}+\partial^{\mu} W\left[J, K_{0}, \vec{K}\right]_{J_{a}^{\mu} K_{c}} \Gamma\left[J, K_{0}, \vec{\phi}\right]_{\phi_{c} J_{b}^{\nu}} \\
& =-\frac{2}{m_{D}} \Gamma_{\phi_{a} J_{b}^{\nu}}-\frac{4}{m_{D}^{2}} \partial_{\nu} \delta(x-y) \delta_{a b}+\int \frac{2 d^{D} z}{m_{D}} \delta(x-z) \delta_{a c} \Gamma_{\phi_{c}(z) J_{b}^{\nu}} \\
& =-\frac{4}{m_{D}^{2}} \partial_{\nu} \delta(x-y) \delta_{a b}
\end{aligned}
$$

where we have used eqs. (74) and (63). The above equation shows the cancellation of the higher loop contributions. 


\subsection{Feynman rules}

As discussed in Sec. 7 we first construct the amplitudes where only the flat connection and the constrained component $\phi_{0}$ are involved and then we use the functional eqs. (47) and (49) in order to obtain all the other (where also the $\phi_{a}$ appears). In the first step we borrow the Feynman rules of the perturbative path integral in order to exploit the properties that are automatically provided by this scheme as unitarity, correct symmetry factors, etc. That means:

- Vertexes:

$$
i \Gamma_{J_{a}^{\mu}, \ldots, K_{0}, \ldots, \phi_{a}, \ldots}^{(0)}
$$

- Propagator:

$$
-i W_{K_{a} K_{b}}^{(0)}=\frac{i}{p^{2}+i \epsilon} \delta_{a b}
$$

- Integration on internal lines:

$$
\frac{1}{(2 \pi)^{D}} \int d^{D} k
$$

- Symmetry factors.

\section{Solution at the tree level}

In this section we construct some amplitudes at the tree level. This is a redundant labor since we know already a solution of eq. (49) i.e. the classical action (52). Functional derivatives of the classical action give all possible vertexes. Here the aim is to show how the eqs. (47) and (49) can fix completely the solution. We require that at the tree level

$$
\left.\frac{\delta^{n} \Gamma^{(0)}\left[J, K_{0}, \vec{\phi}\right]}{\delta J_{a}^{\mu} \ldots \delta K_{0} \ldots}\right|_{J=K_{0}=\vec{K}=0}=0
$$

whenever there is more than one insertion of composite operators $\left(F_{\mu}\right.$ or $\left.\phi_{0}\right)$. With this Ansatz we try to determine the solution at the tree level. We will find up to four point amplitude that the solution agrees with a naïve reading of the action in eq. (52). 


\subsection{Two-point functions}

From eqs. (62), (63) and (83) we get

$$
\Gamma_{J_{a}^{\mu} \phi_{b}}^{(0)}=-i 2 \frac{p_{\mu}}{m_{D}} \delta_{a b}
$$

and

$$
\Gamma_{\phi_{a} \phi_{b}}^{(0)}=\delta_{a b} p^{2} .
$$

\subsection{Three-point functions}

Take now further derivatives of eq. (49)

$$
\begin{aligned}
& \frac{m_{D}^{2}}{2} \partial^{\mu_{1}} \Gamma_{J_{a_{1}}^{\mu_{1}} \phi_{a_{2}} \phi_{a_{3}}}^{(0)}+m_{D} \Gamma_{\phi_{a_{1}} \phi_{a_{2}} \phi_{a_{3}}}^{(0)} \\
& +g\left(\epsilon_{a_{1} b a_{3}} \Gamma_{\phi_{b} \phi_{a_{2}}}^{(0)} \delta\left(x_{1}-x_{3}\right)-\epsilon_{a_{1} a_{2} b} \Gamma_{\phi_{b} \phi_{a_{3}}}^{(0)} \delta\left(x_{1}-x_{2}\right)\right)=0 . \\
& \frac{m_{D}^{2}}{2} \partial^{\mu_{1}} \Gamma_{J_{a_{1}}^{\mu_{1}} J_{a_{2}}^{\mu_{2} \phi_{a_{3}}}}^{(0)}+m_{D} \Gamma_{\phi_{a_{1}} J_{a_{2}}^{\mu_{2} \phi_{a_{3}}}}^{(0)} \\
& +g \epsilon_{a_{1} b a_{3}} \Gamma_{\phi_{b} J_{a_{2}}^{\mu_{2}}}^{(0)} \delta\left(x_{1}-x_{3}\right)+2 g \epsilon_{a_{1} b a_{2}} \Gamma_{J_{b}^{\mu_{2} \phi_{a_{3}}}}^{(0)} \delta\left(x_{1}-x_{2}\right)=0 . \\
& \frac{m_{D}^{2}}{2} \partial^{\mu_{1}} \Gamma_{J_{a_{1}}^{\mu_{1}} J_{a_{2}}^{\mu_{2}} J_{a_{3}}^{\mu_{3}}}^{(0)}+m_{D} \Gamma_{\phi_{a_{1}} J_{a_{2}}^{\mu_{2}} J_{a_{3}}^{\mu_{3}}}^{(0)} \\
& +2 g \epsilon_{a_{1} b a_{3}} \Gamma_{J_{a_{2}}^{\mu_{2}} J_{b}^{\mu_{3}}}^{(0)} \delta\left(x_{1}-x_{3}\right)+2 g \epsilon_{a_{1} b a_{2}} \Gamma_{J_{b}^{\mu_{2}} J_{a_{3}}^{\mu_{3}}}^{(0)} \delta\left(x_{1}-x_{2}\right)=0 .
\end{aligned}
$$

From eqs. (83) and (65) one gets

$$
\Gamma_{J_{a_{1}}^{\mu_{1} \phi_{a_{2}} \phi_{a_{3}}}}^{(0)}=2 i \frac{g}{m_{D}^{2}} \epsilon_{a_{1} a_{2} a_{3}}\left(p_{2 \mu_{1}}-p_{3 \mu_{1}}\right)
$$

and from eq. (85)

$$
\Gamma_{\phi_{a_{1}} \phi_{a_{2}} \phi_{a_{3}}}^{(0)}=0 .
$$

By means of the 1PI amplitudes (84), (85) and (89) one can easily construct the connected amplitude given in eq. (17).

Finally by differentiating eq. (49) with respect to $K_{0}, \phi_{b}$ we get

$$
\frac{m_{D}^{2}}{2} \partial^{\mu} \Gamma_{J_{a}^{\mu} K_{0} \phi_{b}}^{(0)}+m_{D} \Gamma_{K_{0} \phi_{a} \phi_{b}}^{(0)}+g^{2} \delta_{a b}=0 .
$$


and similarly

$$
\frac{m_{D}^{2}}{2} \partial^{\mu_{1}} \Gamma_{J_{a_{1}}^{\mu_{1}} J_{a_{2}}^{\mu_{2} K_{0}}}^{(0)}+\Gamma_{K_{0} K_{0}}^{(0)} \Gamma_{\phi_{a_{1}} J_{a_{2}}^{\mu_{2}}}^{(0)}+\Gamma_{K_{0}}^{(0)} \Gamma_{\phi_{a_{1}} J_{a_{2}}^{\mu_{2} K_{0}}}^{(0)}=0 .
$$

At the tree level we get

$$
\Gamma_{K_{0} \phi_{a} \phi_{b}}^{(0)}=-\frac{g^{2}}{m_{D}} \delta_{a b} .
$$

\subsection{Four-point functions}

Now we consider four-point functions. We take all possible derivatives of eq. (49). We use some simplification in the notations

$$
\begin{aligned}
& \frac{m_{D}^{2}}{2} \partial^{\mu_{1}} \Gamma_{J_{a_{1}}^{\mu_{1}} \phi_{a_{2}} \phi_{a_{3} \phi_{a_{4}}}^{(0)}}+m_{D} \Gamma_{\phi_{a_{1}} \phi_{a_{2}} \phi_{a_{3}} \phi_{a_{4}}}^{(0)} \\
& +\sum_{j=2}^{4} \Gamma_{K_{0} \phi_{a_{j+1}} \phi_{a_{j+2}}}^{(0)} \Gamma_{\phi_{a_{1}} \phi_{a_{j}}}^{(0)}+\sum_{j=2}^{4} g \epsilon_{a_{1} b a_{j}} \Gamma_{\phi_{b} \phi_{a_{j+1}} \phi_{a_{j+2}}}^{(0)} \delta\left(x_{1}-x_{j}\right)=0 .
\end{aligned}
$$

In the above equation at the tree level the last term should be zero according to eq. (90).

$$
\begin{aligned}
& \frac{m_{D}^{2}}{2} \partial^{\mu_{1}} \Gamma_{J_{a_{1}}^{\mu_{1}} J_{a_{2}}^{\mu_{2} \phi_{a_{3}} \phi_{a_{4}}}}^{(0)}+m_{D} \Gamma_{\phi_{a_{1}} J_{a_{2}}^{\mu_{2} \phi_{a_{3}} \phi_{a_{4}}}}^{(0)} \\
& +\Gamma_{K_{0} \phi_{a_{3}} \phi_{a_{4}}}^{(0)} \Gamma_{\phi_{a_{1}} J_{a_{2}}^{\mu_{2}}}^{(0)}+\sum_{j=3,4}\left(\Gamma_{K_{0} J_{a_{2}}^{\mu_{2} \phi_{a_{j}}}}^{(0)} \Gamma_{\phi_{a_{1}} \phi_{a_{j+1}}}^{(0)}\right. \\
& \left.+g \epsilon_{a_{1} b a_{j}} \Gamma_{\phi_{b} J_{a_{2}}^{\mu_{2} \phi_{a_{j+1}}}}^{(0)} \delta\left(x_{1}-x_{j}\right)\right)+2 g \epsilon_{a_{1} b a_{2}} \Gamma_{J_{b}^{\mu} \phi_{a_{3} \phi_{a_{4}}}^{(0)}}^{(0)} \delta\left(x_{1}-x_{2}\right)=0 .
\end{aligned}
$$

According to the Ansatz in eq. (83) the first term in eq. (170) is zero at the tree level.

$$
\begin{aligned}
& \frac{m_{D}^{2}}{2} \partial^{\mu_{1}} \Gamma_{J_{a_{1}}^{\mu_{1}} J_{a_{2}}^{\mu_{2}} J_{a_{3} \phi_{a_{4}}}^{\mu_{3}}}^{(0)}+m_{D} \Gamma_{\phi_{a_{1}} J_{a_{2}} J_{a_{3}}^{\mu_{3} \phi_{a_{4}}}}^{(0)} \\
& +\Gamma_{K_{0} J_{a_{2}}^{\mu_{2} \phi_{a_{4}}}}^{(0)} \Gamma_{\phi_{a_{1}} J_{a_{3}}^{\mu_{3}}}^{(0)}+\Gamma_{K_{0} J_{a_{3}}^{\mu_{3} \phi_{a_{4}}}}^{(0)} \Gamma_{\phi_{a_{1}} J_{a_{2}}^{\mu_{2}}}^{(0)}
\end{aligned}
$$

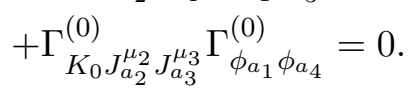

Finally we take only derivatives respect to $J_{a \mu}$

$$
\begin{aligned}
& \frac{m_{D}^{2}}{2} \partial^{\mu_{1}} \Gamma_{J_{a_{1}}^{\mu_{1}} J_{a_{2}}^{\mu_{2}} J_{a_{3}}^{\mu_{3}} J_{a_{4}}^{\mu_{4}}}^{(0)}+m_{D} \Gamma_{\phi_{a_{1}} J_{a_{2}}^{\mu_{2}} J_{a_{3}}^{\mu_{3}} J_{a_{4}}^{\mu_{4}}}^{(0)} \\
& +\sum_{j=2}^{4} \Gamma_{K_{0} J_{a_{j+1}}^{\mu_{j+1}} J_{a_{j+2}}^{\mu_{j+2}}}^{(0)} \Gamma_{\phi_{a_{1}} J_{a_{j}}^{\mu_{j}}}^{(0)}+2 g \sum_{j=2}^{4} \epsilon_{a_{1} b a_{j}} \Gamma_{J_{b}^{\mu_{j}} J_{a_{j+1}}^{\mu_{j+1}} J_{a_{j+2}}^{\mu_{j+2}}}^{(0)} \delta\left(x_{1}-x_{j}\right)=0 .
\end{aligned}
$$


From eq. (95) we get

$$
\begin{aligned}
& \Gamma_{J_{a_{1}}^{\mu_{1}} \phi_{a_{2}} \phi_{a_{3}} \phi_{a_{4}}}^{(0)}=-i 2 \frac{g^{2}}{m_{D}^{3}}\left(\left(p_{1}+2 p_{2}\right)_{\mu_{1}} \delta_{a_{3} a_{4}} \delta_{a_{1} a_{2}}\right. \\
& \left.+\left(p_{1}+2 p_{3}\right)_{\mu_{1}} \delta_{a_{2} a_{4}} \delta_{a_{1} a_{3}}+\left(p_{1}+2 p_{4}\right)_{\mu_{1}} \delta_{a_{2} a_{3}} \delta_{a_{1} a_{4}}\right) .
\end{aligned}
$$

and from eq. (94)

$$
\begin{aligned}
& \Gamma_{\phi_{a_{1}} \phi_{a_{2}} \phi_{a_{3}} \phi_{a_{4}}}^{(0)}=\frac{g^{2}}{m_{D}^{2}}\left(\delta_{a_{3} a_{4}} \delta_{a_{1} a_{2}}\left(p_{2}+p_{1}\right)^{2}\right. \\
& \left.+\delta_{a_{2} a_{4}} \delta_{a_{1} a_{3}}\left(p_{3}+p_{1}\right)^{2}+\delta_{a_{2} a_{3}} \delta_{a_{1} a_{4}}\left(p_{4}+p_{1}\right)^{2}\right) .
\end{aligned}
$$

Eq. (99) says that the flat connection indeed describes a scalar particle which interacts with itself.

From the brief analysis performed up to now one sees that there is a natural hierarchy in the equations. From the functions with the highest number of fields of the flat connection and the constrained component $\phi_{0}$ one derives those where the scalar field is involved.

\section{Reconstruction of the connected amplitudes}

The connected amplitudes can be constructed in a straightforward way by using the obtained results for the 1PI amplitudes. The starting point is given by the relations in eqs. (48). By using the chain rules for the functional derivatives respect to $J_{a \mu}$ with fixed $K_{a}$

$$
\frac{\delta \phi_{z}}{\delta J_{1}}=W_{J_{1} K_{z}}=\Gamma_{J_{1} \phi_{w}} W_{K_{w} K_{z}}=-\Gamma_{J_{1} \phi_{w}}\left[\Gamma_{\phi_{w} \phi_{z}}\right]^{-1}
$$

and (see eq. (788)

$$
\begin{aligned}
& W_{J_{a}^{\mu} J_{b}^{\nu}}=\Gamma_{J_{a}^{\mu} J_{b}^{\nu}}+\Gamma_{J_{a}^{\mu} \phi_{c}} \frac{\delta \phi_{c}}{\delta J_{b}^{\nu}} \\
& =\Gamma_{J_{a}^{\mu} J_{b}^{\nu}}+\Gamma_{J_{a}^{\mu} \phi_{c}} W_{K_{c} K_{w}} \Gamma_{\phi_{w} J_{b}^{\nu}}
\end{aligned}
$$

A further derivative respect to $J_{a \mu}$ with fixed $K_{a}$ is straightforward. The only new quantity is

$$
\frac{\delta}{\delta J_{a}^{\mu}} W_{K_{c} K_{w}}=\Gamma_{J_{a}^{\mu} K_{c} K_{w}}=\Gamma_{J_{a}^{\mu} \phi_{c^{\prime}} \phi_{w^{\prime}}} W_{K_{c^{\prime}} K_{c}} W_{K_{w^{\prime}} K_{w}} .
$$


Thus we have simple rules in order to construct the full connected amplitude with $n+1$ external legs of the flat connection:

1. Start form the n-derivative of $W$ where all the amplitudes are expressed in terms of 1PI functions, with the exception of the $\phi-\phi$ propagator for which it is convenient to use the connected amplitude.

2. Perform the derivative respect to $J_{a \mu}$ with fixed $K_{a}$ and put subsequently all the external sources to zero. To this purpose the 1PI amplitudes are necessary, together with the identities in eqs. (100)) and (102) ).

3. For the final expression, it is convenient to perform the replacement (see eq. (100)

$$
\Gamma_{J_{1} \phi_{w}} W_{K_{w} K_{z}} \rightarrow W_{J_{1} K_{z}} .
$$

\section{One-loop}

The radiative corrections to the tree level amplitudes is the main problem to be solved. There are few items to be discussed. First of all we need a recipe for the construction of the flat connection amplitudes, i.e. those that are at the top of the hierarchy. We use the Feynman rules introduced in Section 8 and neglect all graphs containing tadpoles. We will show that this recipe gives a solution of the functional equation in $\mathrm{D}$ dimensions at the one-loop level. In order to show this we use the identities at the tree level as in Section 9 on the integrands. The examples of the present Section and of Section 12 demonstrate that indeed up to two loops the functional identities (47) and (49) are satisfied by neglecting the tadpoles. In Section 13] it is shown that for higher loop amplitudes tha proof of the validity of the functional equations is more complex and necessitate the use of the eq. (47) at the tree level. The second important item is how we extract a finite part from a divergent amplitude when the limit $D \rightarrow 4$ is taken. We use dimensional subtraction. This procedure is rather subtle since it might violate the functional eqs. (47) and (49). The validity of functional equations is guaranteed if the local counterterms satisfy eq. (58). In particular one has to fix first the counterterms for the amplitudes involving only the flat connection and the constrained component $\phi_{0}$. The pole subtraction has to be performed on the amplitudes normalized according to eq. (50) as discussed in Section 6 , In Section 14 we illustrate this strategy.

Let us start from the one-loop correction of the two-point function of the flat connection. 


\subsection{Two-point amplitudes}

Let us first consider the one-loop correction to the eq. (62). The most straightforward way to perform this is to "close" two $\phi$-lines in eq. (69). From eq. (89) we get

$$
\begin{aligned}
& \Gamma_{J_{a_{1}}^{\mu_{1}} J_{a_{1}^{\prime}}^{\mu_{1}^{\prime}}}^{(1)}\left(p_{1}\right)=-i \delta_{a_{1} a_{1}^{\prime}} \frac{(2 g)^{2}}{m_{D}^{4}} \int \frac{d^{D} p_{2}}{(2 \pi)^{D}} \frac{\left(2 p_{2}+p_{1}\right)_{\mu_{1}}\left(2 p_{2}+p_{1}\right)_{\mu_{1}^{\prime}}}{p_{2}^{2}\left(p_{1}+p_{2}\right)^{2}} \\
& =i \frac{(2 g)^{2}}{m_{D}^{4}} \frac{\delta_{a_{1} a_{1}^{\prime}}}{D-1} \frac{\Gamma\left(2-\frac{D}{2}\right)\left[\Gamma\left(\frac{D}{2}-1\right)\right]^{2}}{(4 \pi)^{\frac{D}{2}} \Gamma(D-2)} \\
& \frac{i}{\left(-p_{1}^{2}\right)^{2-\frac{D}{2}}}\left(p_{1}^{2} g_{\mu_{1} \mu_{1}^{\prime}}-p_{1 \mu_{1}} p_{1 \mu_{1}^{\prime}}\right) \\
& =i \frac{(2 g)^{2}}{m_{D}^{4}} \frac{\delta_{a_{1} a_{1}^{\prime}}}{D-1}\left(p_{1}^{2} g_{\mu_{1} \mu_{1}^{\prime}}-p_{1 \mu_{1}} p_{1 \mu_{1}^{\prime}}\right) I_{2}\left(p_{1}\right),
\end{aligned}
$$

where $I_{2}$ is defined in eq. (154). With the same procedure one obtains

$$
\begin{aligned}
& \Gamma_{J_{a}^{\mu} \phi_{b}}^{(1)}=0 \\
& \Gamma_{\phi_{a} \phi_{b}}^{(1)}=0 .
\end{aligned}
$$

The vertex functional for the $K_{0}$ two-point function is given by

$$
\begin{aligned}
& i \frac{g^{4}}{8 m_{D}^{2}} \int d^{D} x d^{D} y K_{0}(x)\left(\phi^{2}(x) \phi^{2}(y)\right)_{+} K_{0}(y) \\
& =-i \frac{3 g^{4}}{4 m_{D}^{2}} \int d^{D} x d^{D} y K_{0}(x) I_{2}(x-y) K_{0}(y) .
\end{aligned}
$$

\subsection{Three-point amplitudes}

The set of graphs involved at one loop level for the three-point amplitudes are given in Fig. 2, Again we follow the hierarchy of the set of eqs. (64)66). The four-divergence on the vertex functions generates some tadpole graphs. By putting them equal zero the equation (49) is satisfied. We shall illustrate this at the level of integrand since we work in $D$ dimensions. The starting point is then the three-point function of the flat-connection.

$$
\begin{aligned}
& \Gamma_{J_{a_{1}}^{\mu_{1}} J_{a_{2}}^{\mu_{2}} J_{a_{3}}^{\mu_{3}}}^{(1)}\left(p_{1}, p_{2}, p_{3}\right)=i \int \frac{d^{D} k}{(2 \pi)^{D}} \Gamma_{J_{a_{1}}^{\mu_{1}} \phi_{b} \phi_{c}}^{(0)}\left(p_{1}, k,-p_{1}-k\right) \\
& \Gamma_{J_{a_{2}}^{\mu_{2} \phi_{c} \phi_{c^{\prime}}}}^{(0)}\left(p_{2}, p_{1}+k, p_{3}-k\right) \Gamma_{J_{a_{3}}^{\mu_{3} \phi_{c^{\prime}} \phi_{b}}}^{(0)}\left(p_{3},-p_{3}+k,-k\right) \\
& \frac{1}{k^{2}\left(p_{1}+k\right)^{2}\left(p_{3}-k\right)^{2}}
\end{aligned}
$$



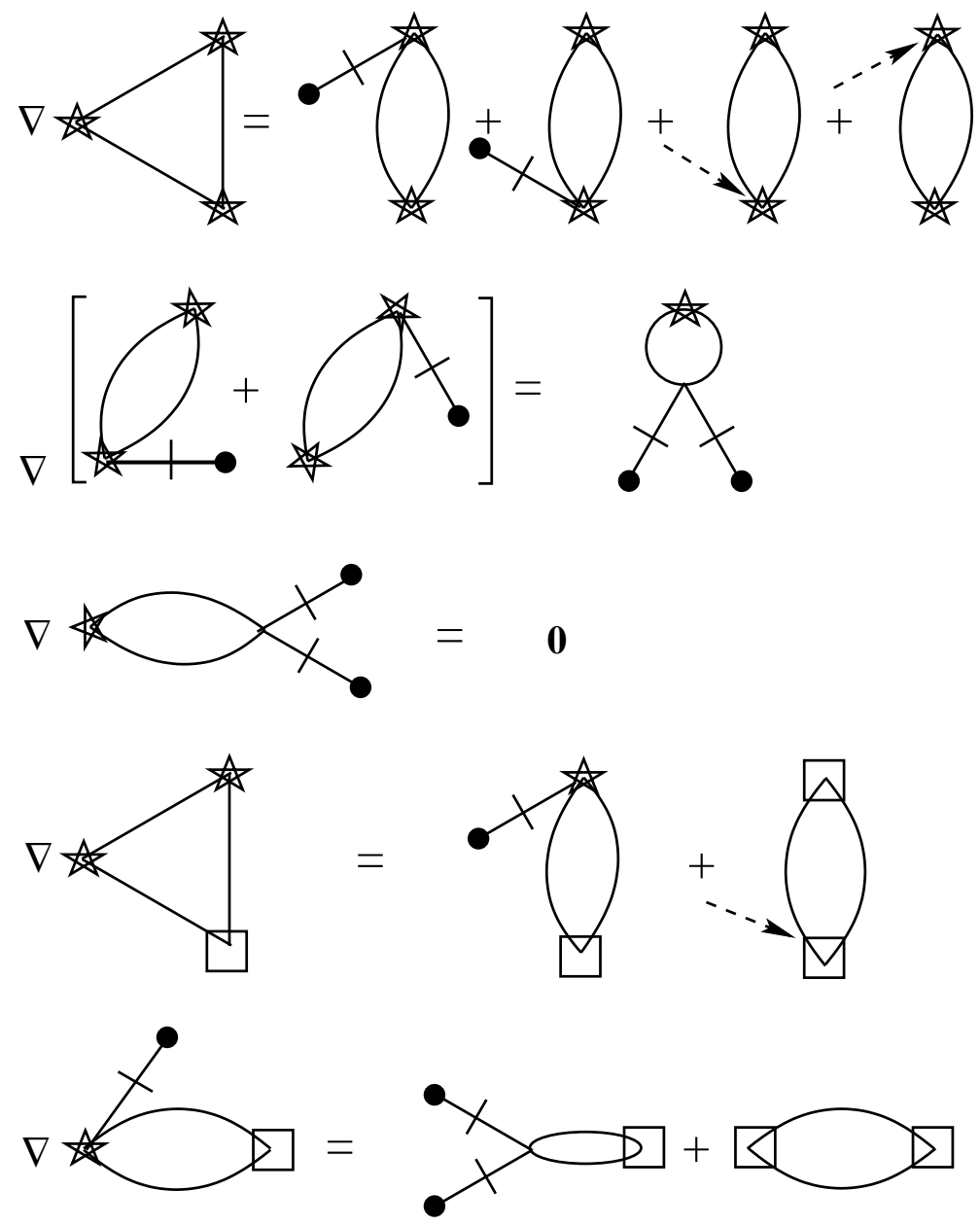

Figure 2: Schematic representation of eq. (49) for the three-point functions in the one-loop approximation. The meaning of the symbols is: $\nabla=i p_{1}^{\mu_{1}}$, star $=$ flat-connection, circle $=\phi_{a}, \square=\phi_{0}$, dashed arrow $=$ flow of external momentum, cut line $=$ amputated $\phi_{a}$-propagator . 
Now we check the eq. (86)

$$
\begin{aligned}
& -i \frac{m_{D}^{2}}{2} p^{\mu_{1}} \Gamma_{J_{a_{1}}^{\mu_{1}} J_{a_{2}}^{\mu_{2}} J_{a_{3}}^{\mu_{3}}\left(p_{1}, p_{2}, p_{3}\right)} \\
& =-\frac{i}{2} \frac{g}{(2 \pi)^{D}} \int d^{D} k \epsilon_{a_{1} b c}\left[\frac{1}{\left(p_{1}+k\right)^{2}\left(p_{3}-k\right)^{2}}-\frac{1}{k^{2}\left(p_{3}-k\right)^{2}}\right] \\
& \frac{(2 i g)^{2}}{m_{D}^{4}}\left(\delta_{a_{3} c} \delta_{a_{2} b}-\delta_{a_{2} a_{3}} \delta_{b c}\right)\left(p_{1}-p_{3}+2 k\right)_{\mu_{2}}\left(-p_{3}+2 k\right)_{\mu_{3}} \\
& =\frac{i}{2} \frac{(2 g)^{3}}{m_{D}^{4}} \int \frac{d^{D} k}{(2 \pi)^{D}} \epsilon_{a_{1} a_{2} a_{3}}\left[\frac{1}{\left(p_{1}+k\right)^{2}\left(p_{3}-k\right)^{2}}-\frac{1}{k^{2}\left(p_{3}-k\right)^{2}}\right] \\
& \left(p_{1}-p_{3}+2 k\right)_{\mu_{2}}\left(-p_{3}+2 k\right)_{\mu_{3}} \\
& =\frac{i}{2} \frac{(2 g)^{3}}{m_{D}^{4}} \int \frac{d^{D} k}{(2 \pi)^{D}} \epsilon_{a_{1} a_{2} a_{3}}\left[\frac{\left(2 k-p_{2}+p_{1}\right)_{\mu_{2}}\left(2 k-p_{2}\right)_{\mu_{3}}}{k^{2}\left(p_{2}-k\right)^{2}}\right. \\
& -\frac{\left(2 k-p_{3}+p_{1}\right)_{\mu_{2}}\left(2 k-p_{3}\right)_{\mu_{3}}}{k^{2}\left(p_{3}-k\right)^{2}} .
\end{aligned}
$$

The equation (66) involve also the expression in eq. (104) and

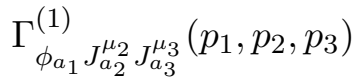

$$
\begin{aligned}
& =\frac{i}{2} \frac{(2 g)^{3}}{m_{D}^{5}} \int \frac{d^{D} k}{(2 \pi)^{D}} \epsilon_{a_{1} a_{2} a_{3}}\left[\frac{\left(2 k-p_{2}\right)_{\mu_{2}}\left(2 k-p_{2}\right)_{\mu_{3}}}{k^{2}\left(p_{2}-k\right)^{2}}\right. \\
& \left.-\frac{\left(2 k-p_{3}\right)_{\mu_{2}}\left(2 k-p_{3}\right)_{\mu_{3}}}{k^{2}\left(p_{3}-k\right)^{2}}\right] \text {. }
\end{aligned}
$$

By using eq. (104) we get

$$
\begin{aligned}
& =-\frac{i}{2} \frac{(2 g)^{3}}{m^{5}} \frac{1}{D-1} \epsilon_{a_{1} a_{2} a_{3}}\left[\left(p_{2}^{2} g_{\mu_{2} \mu_{3}}-p_{2 \mu_{2}} p_{2 \mu_{3}}\right) I_{2}\left(p_{2}\right)\right. \\
& \left.-\left(p_{3}^{2} g_{\mu_{2} \mu_{3}}-p_{3 \mu_{2}} p_{3 \mu_{3}}\right) I_{2}\left(p_{3}\right)\right]
\end{aligned}
$$

Then one can easily verify eq. (66) by using eq. (104)

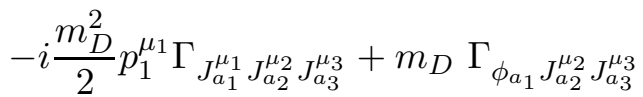

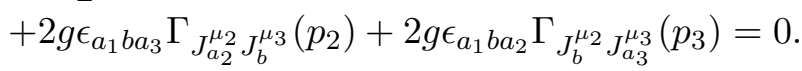

Now we consider eq. (65). We need the one loop correction $\Gamma_{J_{a_{1}} \phi_{a_{2}} \phi_{a_{3}}}^{(1)}$ :

$$
\begin{aligned}
& \Gamma_{\phi_{a_{1}} J_{a_{2}}^{\mu_{2} \phi_{a_{3}}}}^{(1)}\left(p_{1}, p_{2}, p_{3}\right) \\
& =\frac{g^{3}}{m_{D}^{4}} \int \frac{d^{D} k}{(2 \pi)^{D}} \epsilon_{a_{1} a_{2} a_{3}}\left(p_{1}-p_{3}\right)^{\mu_{1}}\left[\frac{\left(2 k+p_{2}\right)_{\mu_{1}}\left(2 k+p_{2}\right)_{\mu_{2}}}{k^{2}\left(p_{2}+k\right)^{2}}\right]
\end{aligned}
$$


and again by neglecting tadpole graphs the functional equation (65) is satisfied. Equation (64) is simply

$$
p_{1}^{\mu} \Gamma_{J_{a_{1}}^{\mu} \phi_{a_{2}} \phi_{a_{3}}}^{(1)}=0 .
$$

We evaluate the amplitude $\Gamma_{J \phi \phi}^{(1)}$ in coordinate space

$$
i \frac{g^{3}}{m_{D}^{4}} \int d^{D} x \int d^{D} y J_{a_{1}}^{\mu}(x)\left(\epsilon_{a_{1} a b} \partial_{\mu} \phi_{a} \phi_{b}(x) \quad \phi_{c} \partial_{\nu} \phi_{c} \phi_{d} \partial^{\nu} \phi_{d}(y)\right)_{+}
$$

The relevant vertex function is

$$
\begin{aligned}
& \Gamma_{J \phi \phi}^{(1)}=-i \frac{g^{3}}{m_{D}^{4}} \int d^{D} x \int d^{D} y J_{c}^{\mu}(x) \epsilon_{a b c} \\
& \frac{1}{(D-1)}\left(\square g_{\mu \nu}-\partial_{\mu} \partial_{\nu}\right) I_{2}(x-y) \phi_{a} \partial^{\nu} \phi_{b}(y) .
\end{aligned}
$$

Finally we have the last equation (67) involving three-point functions. Thus we need

$$
\begin{aligned}
& \Gamma_{J_{a_{1}}^{\mu_{1} \phi_{a_{2}} K_{0}}}^{(1)}\left(p_{1}, p_{2}, p_{3}\right)=\frac{g^{4}}{m_{D}^{4}} \int \frac{d^{D} k}{(2 \pi)^{D}} \\
& \left(3 p_{1}+4 p_{2}\right)_{\mu_{1}} \frac{1}{k^{2}\left(p_{3}-k\right)^{2}},
\end{aligned}
$$

which we now evaluate in coordinate space:

$$
\begin{aligned}
& i \frac{g^{4}}{2 m_{D}^{4}} \int d^{D} x \int d^{D} y\left[\left(2 J_{a_{1}}^{\mu} \phi^{2}(x) \partial_{\mu} \phi_{a_{1}}(x)\right.\right. \\
& \left.\left.+\partial_{\mu} J_{a_{1}}^{\mu} \phi^{2}(x) \phi_{a_{1}}(x)\right) \phi^{2}(y)\right]_{+} K_{0}(y)
\end{aligned}
$$

The final result is

$$
\begin{aligned}
& i \frac{g^{4}}{2 m_{D}^{4}} \int d^{D} x \int d^{D} y\left[-12 J_{a_{1}}^{\mu} \partial_{\mu} \phi_{a_{1}}(x)+4 \partial_{\mu}\left(J_{a_{1}}^{\mu} \phi_{a_{1}}\right)\right. \\
& \left.-10 \partial_{\mu} J_{a_{1}}^{\mu} \phi_{a_{1}}(x)\right] I_{2}(x-y) K_{0}(y) \\
& =-i \frac{g^{4}}{m_{D}^{4}} \int d^{D} x \int d^{D} y\left[4 J_{a_{1}}^{\mu} \partial_{\mu} \phi_{a_{1}}+3 \partial_{\mu} J_{a_{1}}^{\mu} \phi_{a_{1}}\right](x) \\
& I_{2}(x-y) K_{0}(y) .
\end{aligned}
$$

We need a further amplitude

$$
\begin{aligned}
& \Gamma_{\phi_{a_{1}} \phi_{a_{2}} K_{0}}^{(1)}\left(p_{1}, p_{2}, p_{3}\right)=i \frac{g^{4}}{4 m_{D}^{3}} \int \frac{d^{D} k}{(2 \pi)^{D}} \\
& \left(4\left(p_{1}^{2}+p_{2}^{2}\right)+6 p_{1} p_{2}-2 k p_{3}\right) \frac{1}{k^{2}\left(p_{3}-k\right)^{2}} \\
& =i \frac{g^{4}}{4 m_{D}^{3}} \int \frac{d^{D} k}{(2 \pi)^{D}}\left(3\left(p_{1}^{2}+p_{2}^{2}\right)+4 p_{1} p_{2}\right) \frac{1}{k^{2}\left(p_{3}-k\right)^{2}} .
\end{aligned}
$$


We explicitly evaluate the vertex functional relevant for $\Gamma_{K_{0} \phi \phi}^{(1)}$. We need the contractions on

$$
-i \frac{g^{4}}{4 m_{D}^{3}} \int d^{D} x d^{D} y K_{0}(x)\left(\phi(x)^{2} \quad \phi_{a} \partial^{\mu} \phi_{a} \phi_{b} \partial_{\mu} \phi_{b}(y)\right)_{+} .
$$

The relevant functional is

$$
-i \frac{g^{4}}{4 m_{D}^{3}} \int d^{D} x d^{D} y K_{0}(x) I_{2}(x-y)\left(3 \square\left(\phi^{2}\right)(y)-2 \partial^{\mu} \phi_{b} \partial_{\mu} \phi_{b}(y)\right)
$$

which together with the amplitudes in eqs. (118) and (106) satisfy eq. (49).

\subsection{Four-point amplitudes}

It is of some interest to evaluate some four-point amplitudes, since on this point the first serious difficulties of the nonlinear sigma model have been shown [19], [20]. We perform the complete evaluation of the amplitudes involving up to one flat connection insertion. We use the same recipe as before(consisting in the use of tree level Feynman rules and in neglecting tadpole graphs) and we evaluate the amplitudes $\Gamma_{J \phi \phi \phi}$ and $\Gamma_{\phi \phi \phi \phi}$. We find convenient to work in coordinate space. From this results can get also the counterterms and thus check eq. (55).

\section{$11.4 \Gamma_{J \phi \phi \phi}$}

We perform the necessary contractions on the second order perturbative expansion of

$$
-i \frac{g^{4}}{2 m_{D}^{5}} \int d^{D} x \int d^{D} y\left[\left(2 J_{a}^{\mu} \phi^{2} \partial_{\mu} \phi_{a}+\partial_{\mu} J_{a}^{\mu} \phi^{2} \phi_{a}\right)(x)\left(\phi_{c} \partial^{\nu} \phi_{c} \phi_{d} \partial_{\nu} \phi_{d}\right)(y)\right]_{+} .
$$

After some lengthy and straightforward algebra we get

$$
\begin{aligned}
\Gamma^{(1)}[J \phi \phi \phi] \quad & -i \frac{g^{4}}{m_{D}^{5}} \int d^{D} x \int d^{D} y\left\{\frac{1}{2} J_{a}^{\mu} \partial_{\mu} \phi_{a}(x) I_{2}(x-y) \square \phi^{2}(y)\right. \\
& -J_{a}^{\mu} \partial_{\mu} \phi_{a}(x) I_{2}(x-y) \partial_{\nu} \phi_{d} \partial^{\nu} \phi_{d}(y) \\
& +J_{a}^{\mu} \phi_{a}(x) I_{2}(x-y) \partial_{\mu}\left(\partial_{\nu} \phi_{d} \partial^{\nu} \phi_{d}\right)(y) \\
& -\frac{3}{2} J_{a}^{\mu} \phi_{a}(x) I_{2}(x-y) \partial_{\mu} \square \phi^{2}(y) \\
& -\frac{2}{(D-1)} J_{a}^{\mu} \phi_{c}(x) I_{2}(x-y) \quad\left(\square g_{\mu \nu}-\partial_{\mu} \partial_{\nu}\right)\left(\partial^{\nu} \phi_{c} \phi_{a}\right)(y) \\
& \left.+2 J_{a}^{\mu} \partial_{\mu} \phi_{c}(x) I_{2}(x-y) \partial^{\nu} \phi_{a} \partial_{\nu} \phi_{c}(y)\right\}
\end{aligned}
$$


Now we take the derivative in order to check eq. (49).

$$
\begin{aligned}
& \frac{\delta}{\delta J_{a}^{\mu}} \Gamma^{(1)}[J \phi \phi \phi]=-i \frac{g^{4}}{m_{D}^{5}} \int d^{D} y \quad\left\{\frac{1}{2} \square \phi_{a} I_{2}(x-y) \square \phi^{2}+\frac{1}{2} \partial^{\mu} \phi_{a} I_{2}(x-y) \partial_{\mu} \square \phi^{2}\right. \\
& -\square \phi_{a} I_{2}(x-y) \quad \partial_{\nu} \phi_{d} \partial^{\nu} \phi_{d}+\phi_{a} I_{2}(x-y) \quad \square\left(\partial_{\nu} \phi_{d} \partial^{\nu} \phi_{d}\right) \\
& -\frac{3}{2} \partial^{\mu} \phi_{a} I_{2}(x-y) \partial_{\mu} \square \phi^{2}-\frac{3}{2} \phi_{a} I_{2}(x-y) \square^{2} \phi^{2} \\
& -\frac{2}{(D-1)} \partial^{\mu} \phi_{c} I_{2}(x-y) \quad\left(\square g_{\mu \nu}-\partial_{\mu} \partial_{\nu}\right)\left(\partial^{\nu} \phi_{c} \phi_{a}\right) \\
& \left.+2 \square \phi_{c} I_{2}(x-y) \quad \partial^{\nu} \phi_{a} \partial_{\nu} \phi_{c}+2 \partial^{\mu} \phi_{c} I_{2}(x-y) \partial_{\mu}\left(\partial^{\nu} \phi_{a} \partial_{\nu} \phi_{c}\right)\right\} .
\end{aligned}
$$

i.e. we can obtain the first term in eq. (49)

$$
\begin{aligned}
& \frac{m_{D}^{2}}{2} \partial^{\mu} \frac{\delta}{\delta J_{a}^{\mu}} \Gamma_{J \phi \phi \phi}=-i \frac{g^{4}}{2 m_{D}^{3}} \int d^{D} y\left\{\frac{1}{2} \square \phi_{a} I_{2}(x-y) \square \phi^{2}\right. \\
& -\square \phi_{a} I_{2}(x-y) \partial_{\nu} \phi_{d} \partial^{\nu} \phi_{d}+\phi_{a} I_{2}(x-y) \quad \square\left(\partial_{\nu} \phi_{d} \partial^{\nu} \phi_{d}\right) \\
& -\partial^{\mu} \phi_{a} I_{2}(x-y) \partial_{\mu} \square \phi^{2}-\frac{3}{2} \phi_{a} I_{2}(x-y) \square^{2} \phi^{2} \\
& -\frac{2}{(D-1)} \partial^{\mu} \phi_{c} I_{2}(x-y) \quad\left(\square g_{\mu \nu}-\partial_{\mu} \partial_{\nu}\right)\left(\partial^{\nu} \phi_{c} \phi_{a}\right) \\
& \left.+2 \square \phi_{c} I_{2}(x-y) \partial^{\nu} \phi_{a} \partial_{\nu} \phi_{c}+2 \partial^{\mu} \phi_{c} I_{2}(x-y) \partial_{\mu}\left(\partial^{\nu} \phi_{a} \partial_{\nu} \phi_{c}\right)\right\} .
\end{aligned}
$$

\section{$11.5 \Gamma_{\phi \phi \phi \phi}$}

We perform the necessary contractions on the second order perturbative expansion of

$$
i \frac{1}{2}\left(\frac{g^{2}}{2 m_{D}^{2}}\right)^{2} \int d^{D} x d^{D} y\left(\phi_{a} \partial^{\mu} \phi_{a} \phi_{b} \partial_{\mu} \phi_{b}(x) \phi_{c} \partial^{\nu} \phi_{c} \phi_{d} \partial_{\nu} \phi_{d}(y)\right) .
$$

After some algebra one gets

$$
\begin{aligned}
& i\left(\frac{g^{2}}{2 m_{D}^{2}}\right)^{2} \int d^{D} x d^{D} y \\
& \left\{-\frac{3}{4} \square \phi^{2}(x) I_{2}(x-y) \square \phi^{2}(y)+\square \phi^{2}(x) I_{2}(x-y) \partial^{\nu} \phi_{c} \partial_{\nu} \phi_{c}(y)\right. \\
& -\partial^{\mu} \phi_{c} \partial_{\mu} \phi_{b}(x) I_{2}(x-y) \partial^{\nu} \phi_{c} \partial_{\nu} \phi_{b}(y) \\
& \left.-\frac{1}{(D-1)} \partial^{\mu} \phi_{a} \phi_{b}(x) \quad\left(\square g_{\mu \nu}-\partial_{\mu} \partial_{\nu}\right) I_{2}(x-y) \partial_{\nu} \phi_{a} \phi_{b}(y)\right\} .
\end{aligned}
$$


The singular part at $D=4$ of the expression in eq. (127) agrees with the results of Reference [20]. One simply uses the identities

$$
\begin{aligned}
& \square \phi^{2} \square \phi^{2}=4\left[\left(\phi_{a} \square \phi_{a}\right)^{2}+2 \phi_{a} \square \phi_{a} \partial_{\mu} \phi_{b} \partial^{\mu} \phi_{b}+\partial_{\nu} \phi_{a} \partial^{\nu} \phi_{a} \partial_{\mu} \phi_{b} \partial^{\mu} \phi_{b}\right] \\
& \square \phi^{2} \partial_{\nu} \phi_{a} \partial^{\nu} \phi_{a}=2\left[\partial_{\nu} \phi_{a} \partial^{\nu} \phi_{a} \partial_{\mu} \phi_{b} \partial^{\mu} \phi_{b}+\phi_{a} \square \phi_{a} \partial_{\mu} \phi_{b} \partial^{\mu} \phi_{b}\right] \\
& \int d^{D} x \partial^{\mu} \phi_{a} \phi_{b} \quad\left(\square g_{\mu \nu}-\partial_{\mu} \partial_{\nu}\right)\left(\partial^{\nu} \phi_{a} \phi_{b}\right) \\
& =-\int d^{D} x\left[\partial_{\nu}\left(\partial^{\mu} \phi_{a} \phi_{b}\right) \partial^{\nu}\left(\partial_{\mu} \phi_{a} \phi_{b}\right)-\partial_{\nu}\left(\partial^{\mu} \phi_{a} \phi_{b}\right) \partial_{\mu}\left(\partial^{\nu} \phi_{a} \phi_{b}\right)\right] \\
& =\int d^{D} x\left[\partial_{\nu} \phi_{a} \partial^{\nu} \phi_{b} \partial_{\mu} \phi_{a} \partial^{\mu} \phi_{b}-\partial_{\nu} \phi_{a} \partial^{\nu} \phi_{a} \partial_{\mu} \phi_{b} \partial^{\mu} \phi_{b}\right] .
\end{aligned}
$$

In order to check eq. (49) we take the derivative with respect to $\phi_{a}$

$$
\begin{aligned}
& m_{D} \Gamma_{\phi_{a}}=i \frac{g^{4}}{4 m_{D}^{3}} \int d^{D} y\left\{-3 \phi_{a} I_{2}(x-y) \square^{2} \phi^{2}\right. \\
& +2 \phi_{a} I_{2}(x-y) \square\left(\partial^{\nu} \phi_{c} \partial_{\nu} \phi_{c}\right)-2 \square \phi_{a} I_{2}(x-y) \square \phi^{2}-2 \partial_{\nu} \phi_{a} I_{2}(x-y) \partial^{\nu} \square \phi^{2} \\
& +4 \square \phi_{b} I_{2}(x-y) \partial^{\nu} \phi_{a} \partial_{\nu} \phi_{b}+4 \partial_{\mu} \phi_{b} I_{2}(x-y) \partial^{\mu}\left(\partial^{\nu} \phi_{a} \partial_{\nu} \phi_{b}\right) \\
& -\frac{2}{(D-1)} \partial^{\mu} \phi_{c}\left(\square g_{\mu \nu}-\partial_{\mu} \partial_{\nu}\right) I_{2}(x-y)\left(\partial_{\nu} \phi_{c} \phi_{a}\right) \\
& \left.+\frac{2}{(D-1)} \partial^{\mu} \phi_{c}\left(\square g_{\mu \nu}-\partial_{\mu} \partial_{\nu}\right) I_{2}(x-y)\left[\partial_{\nu}\left(\phi_{a} \phi_{c}\right)-\phi_{a} \partial_{\nu} \phi_{c}\right]\right\} \\
& =i \frac{g^{4}}{4 m_{D}^{3}} \int d^{D} y\left\{-3 \phi_{a} I_{2}(x-y) \square^{2} \phi^{2}\right. \\
& +2 \phi_{a} I_{2}(x-y) \square\left(\partial^{\nu} \phi_{c} \partial_{\nu} \phi_{c}\right)-2 \square \phi_{a} I_{2}(x-y) \square \phi^{2}-2 \partial_{\nu} \phi_{a} I_{2}(x-y) \partial^{\nu} \square \phi^{2} \\
& +4 \square \phi_{b} I_{2}(x-y) \partial^{\nu} \phi_{a} \partial_{\nu} \phi_{b}+4 \partial_{\mu} \phi_{b} I_{2}(x-y) \partial^{\mu}\left(\partial^{\nu} \phi_{a} \partial_{\nu} \phi_{b}\right) \\
& \left.-\frac{4}{(D-1)} \partial^{\mu} \phi_{c}\left(\square g_{\mu \nu}-\partial_{\mu} \partial_{\nu}\right) I_{2}(x-y)\left(\partial_{\nu} \phi_{c} \phi_{a}\right)\right\} .
\end{aligned}
$$

The quantities in eqs. (125), (129) and (121) do satisfy eq. (49).

\section{Two-loops}

Now we consider some cases at the two-loop level. We use the Feynman rules derived in Sec. 9 .

\subsection{Two-point functions}

We consider the contribution of the graphs depicted in Fig. 3 For the evaluation of the $\phi-\phi$ two-point function we use the Feynman rule in eq. (99).

$$
\Gamma_{\phi_{a_{1}} \phi_{a_{1}^{\prime}}}^{(2)}\left(p_{1}\right)=i \frac{g^{4}}{6 m_{D}^{4}} \int \frac{d^{D} p_{2}}{(2 \pi)^{D}} \frac{d^{D} p_{3}}{(2 \pi)^{D}} \frac{i^{3}}{p_{2}^{2} p_{3}^{2} p_{4}^{2}}
$$



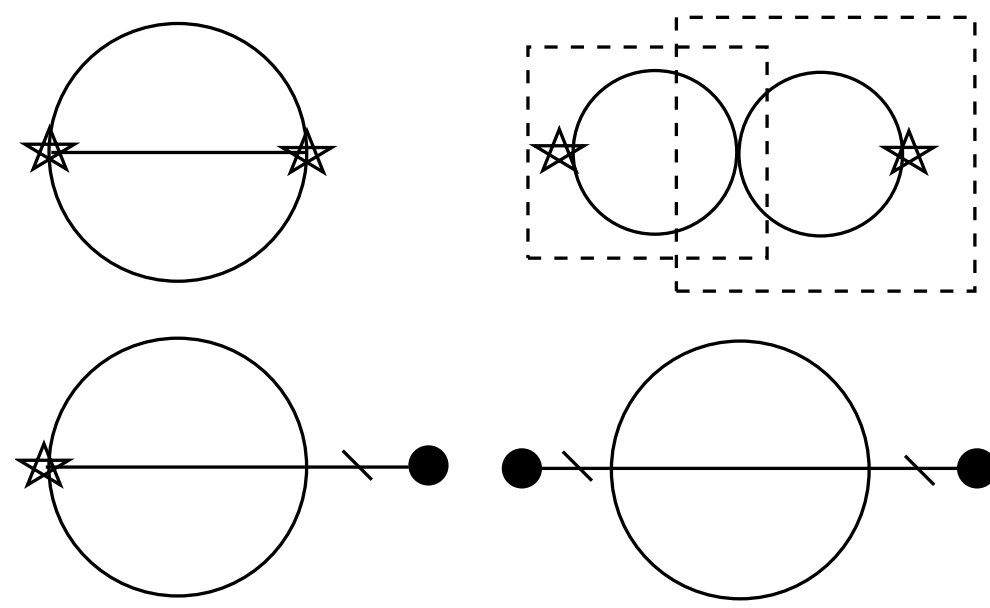

Figure 3: Two-loop graphs for the two-point functions.

$$
\begin{aligned}
& \left(\delta_{a_{3} a_{4}} \delta_{a_{1} a_{2}}\left(p_{2}+p_{1}\right)^{2}\right. \\
& \left.+\delta_{a_{2} a_{4}} \delta_{a_{1} a_{3}}\left(p_{3}+p_{1}\right)^{2}+\delta_{a_{2} a_{3}} \delta_{a_{1} a_{4}}\left(p_{4}+p_{1}\right)^{2}\right) \\
& \left(\delta_{a_{3} a_{4}} \delta_{a_{1}^{\prime} a_{2}}\left(p_{2}+p_{1}\right)^{2}\right. \\
& \left.+\delta_{a_{2} a_{4}} \delta_{a_{1}^{\prime} a_{3}}\left(p_{3}+p_{1}\right)^{2}+\delta_{a_{2} a_{3}} \delta_{a_{1}^{\prime} a_{4}}\left(p_{4}+p_{1}\right)^{2}\right) \\
& =\frac{g^{4}}{6 m_{D}^{4}} \delta_{a_{1} a_{1}^{\prime}} \int \frac{d^{D} p_{2}}{(2 \pi)^{D}} \frac{d^{D} p_{3}}{(2 \pi)^{D}} \frac{1}{p_{2}^{2} p_{3}^{2} p_{4}^{2}} \\
& {\left[9\left[\left(p_{1}+p_{2}\right)^{2}\right]^{2}+6\left(p_{1}+p_{2}\right)^{2}\left(p_{1}+p_{3}\right)^{2}\right]} \\
& =\frac{g^{4}}{6 m_{D}^{4}} \delta_{a_{1} a_{1}^{\prime}} \int \frac{d^{D} p_{2}}{(2 \pi)^{D}} \frac{d^{D} p_{3}}{(2 \pi)^{D}} \frac{1}{p_{2}^{2} p_{3}^{2} p_{4}^{2}}\left\{9 \left[p_{1}^{4}+4\left(p_{1} p_{2}\right)^{2}\right.\right. \\
& \left.\left.+4 p_{1}^{2}\left(p_{1} p_{2}\right)\right]+6\left[p_{1}^{4}+2 p_{1}^{2}\left(p_{1} p_{3}\right)+2 p_{1}^{2}\left(p_{1} p_{2}\right)+4\left(p_{1} p_{2}\right)\left(p_{1} p_{3}\right)\right]\right\}
\end{aligned}
$$

The integration over $p_{3}$ allows to use eq. (155)

$$
\begin{aligned}
& \Gamma_{\phi_{a_{1}} \phi_{a_{1}^{\prime}}}^{(2)}\left(p_{1}\right)=\frac{g^{4}}{6 m_{D}^{4}} \delta_{a_{1} a_{1}^{\prime}}\left\langle\left[15 p_{1}^{4}+48 p_{1}^{2}\left(p_{1} p_{2}\right)+24\left(p_{1} p_{2}\right)^{2}\right]\right\rangle_{3} \\
& =\frac{g^{4}}{6 m_{D}^{4}} \delta_{a_{1} a_{1}^{\prime}} \frac{5 D-4}{3 D-4} I_{3}\left(p_{1}\right) p_{1}^{4} .
\end{aligned}
$$

$I_{3}$ is given in eq. (164). Now we evaluate the next relevant two-point amplitude (the second graph in Figure 3 is not relevant for the check of eq. (63) since it is proportional to a pure transverse tensor)

$$
\Gamma_{J_{a_{1}}^{\mu_{1}} J_{a_{1}^{\prime}}^{\mu^{\prime}}}^{(2)}\left(p_{1}\right)=\frac{i}{6} \int \frac{d^{D} p_{2}}{(2 \pi)^{2 D}} \int \frac{d^{D} p_{3}}{(2 \pi)^{2 D}}
$$




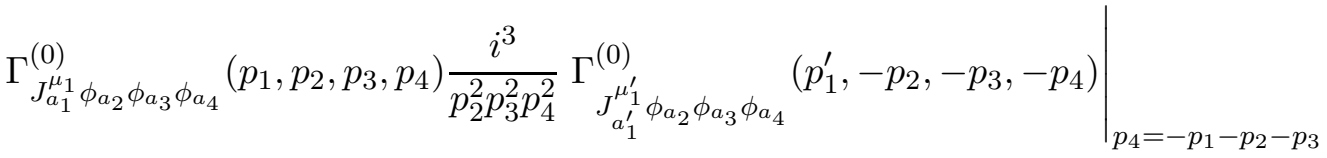

$$
\begin{aligned}
& =\frac{1}{6}\left(\frac{-2 i g^{2}}{m_{D}^{3}}\right)^{2} \int \frac{d^{D} p_{2}}{(2 \pi)^{2 D}} \int \frac{d^{D} p_{3}}{(2 \pi)^{2 D}} \frac{1}{p_{2}^{2} p_{3}^{2} p_{4}^{2}}\left(\delta_{a_{3} a_{4}} \delta_{a_{1} a_{2}}\left(2 p_{2}+p_{1}\right)\right. \\
& \left.+\delta_{a_{2} a_{4}} \delta_{a_{1} a_{3}}\left(2 p_{3}+p_{1}\right)+\delta_{a_{2} a_{3}} \delta_{a_{1} a_{4}}\left(2 p_{4}+p_{1}\right)\right)_{\mu} \\
& \left(\delta_{a_{3} a_{4}} \delta_{a_{1}^{\prime} a_{2}}\left(-2 p_{2}+p_{1}^{\prime}\right)\right. \\
& \left.+\delta_{a_{2} a_{4}} \delta_{a_{1}^{\prime} a_{3}}\left(-2 p_{3}+p_{1}^{\prime}\right)+\delta_{a_{2} a_{3}} \delta_{a_{1}^{\prime} a_{4}}\left(-2 p_{4}+p_{1}^{\prime}\right)\right)_{\mu^{\prime}} \\
& =\frac{1}{6}\left(\frac{2 g^{2}}{m_{D}^{3}}\right)^{2} \int \frac{d^{D} p_{2}}{(2 \pi)^{2 D}} \int \frac{d^{D} p_{3}}{(2 \pi)^{2 D}} \frac{1}{p_{2}^{2} p_{3}^{2} p_{4}^{2}}\left(9\left(2 p_{2}+p_{1}\right)_{\mu}\left(2 p_{2}+p_{1}\right)_{\mu^{\prime}}\right. \\
& \left.+6\left(2 p_{2}+p_{1}\right)_{\mu}\left(2 p_{3}+p_{1}\right)_{\mu^{\prime}}\right) \delta_{a_{1} a_{1}^{\prime}} \text {. }
\end{aligned}
$$

We use the equation (155) for the integration over $p_{3}$

$$
\begin{aligned}
& \Gamma_{J_{a_{1}}^{\mu_{1}} J_{a_{1}^{\prime}}^{\mu^{\prime}}}^{(2)}\left(p_{1}\right) \\
& =\frac{1}{6}\left(\frac{2 g^{2}}{m_{D}^{3}}\right)^{2} \int \frac{d^{D} p_{2}}{(2 \pi)^{2 D}} \int \frac{d^{D} p_{3}}{(2 \pi)^{2 D}} \frac{1}{p_{2}^{2} p_{3}^{2} p_{4}^{2}}\left(9\left(2 p_{2}+p_{1}\right)_{\mu}\left(2 p_{2}+p_{1}\right)_{\mu^{\prime}}\right. \\
& \left.+6\left(2 p_{2}+p_{1}\right)_{\mu}\left(2\left[-\frac{1}{2}\left(p_{1}+p_{2}\right)\right]+p_{1}\right)_{\mu^{\prime}}\right) \delta_{a_{1} a_{1}^{\prime}} \\
& =\frac{1}{6}\left(\frac{2 g^{2}}{m_{D}^{3}}\right)^{2} \int \frac{d^{D} p_{2}}{(2 \pi)^{2 D}} \int \frac{d^{D} p_{3}}{(2 \pi)^{2 D}} \frac{1}{p_{2}^{2} p_{3}^{2} p_{4}^{2}}\left(9 p_{1 \mu} p_{1 \mu^{\prime}}\right. \\
& \left.+18 p_{2 \mu} p_{1 \mu^{\prime}}+12 p_{1 \mu} p_{2 \mu^{\prime}}+p_{2 \mu} p_{2 \mu^{\prime}}\right) \delta_{a_{1} a_{1}^{\prime}} \\
& =\frac{4}{6} \delta_{a_{1} a_{1}^{\prime}}\left(\frac{g^{2}}{m_{D}^{3}}\right)^{2} I_{3}\left(p_{1}\right)\left(-p_{1 \mu} p_{1 \mu^{\prime}}+\frac{8}{3 D-4}\left(-p_{1}^{2} g_{\mu \mu^{\prime}}+D p_{1 \mu} p_{1 \mu^{\prime}}\right)\right) \\
& =\frac{2}{3} \delta_{a_{1} a_{1}^{\prime}}\left(\frac{g^{2}}{m_{D}^{3}}\right)^{2} \frac{I_{3}\left(p_{1}\right)}{3 D-4}\left(-8 p_{1}^{2} g_{\mu \mu^{\prime}}+(5 D+4) p_{1 \mu} p_{1 \mu^{\prime}}\right) .
\end{aligned}
$$

Now we can check the validity of eqs. (62) and (63)

$$
m_{D}^{2} \partial^{\mu} \partial^{\mu^{\prime}} \Gamma_{J_{a_{1}}^{\mu} J_{a_{1}^{\prime}}^{\mu^{\prime}}}-4 \Gamma_{\phi_{a_{1}} \phi_{a_{1}^{\prime}}}=0
$$

\subsection{Three-point functions}

The two-loop three-point functions are drawn in Fig. 4. When we try to check the validity of the relation in eq. (66) we get again some tadpole graphs that we put equal zero. Thus we are left only with the graphs in 

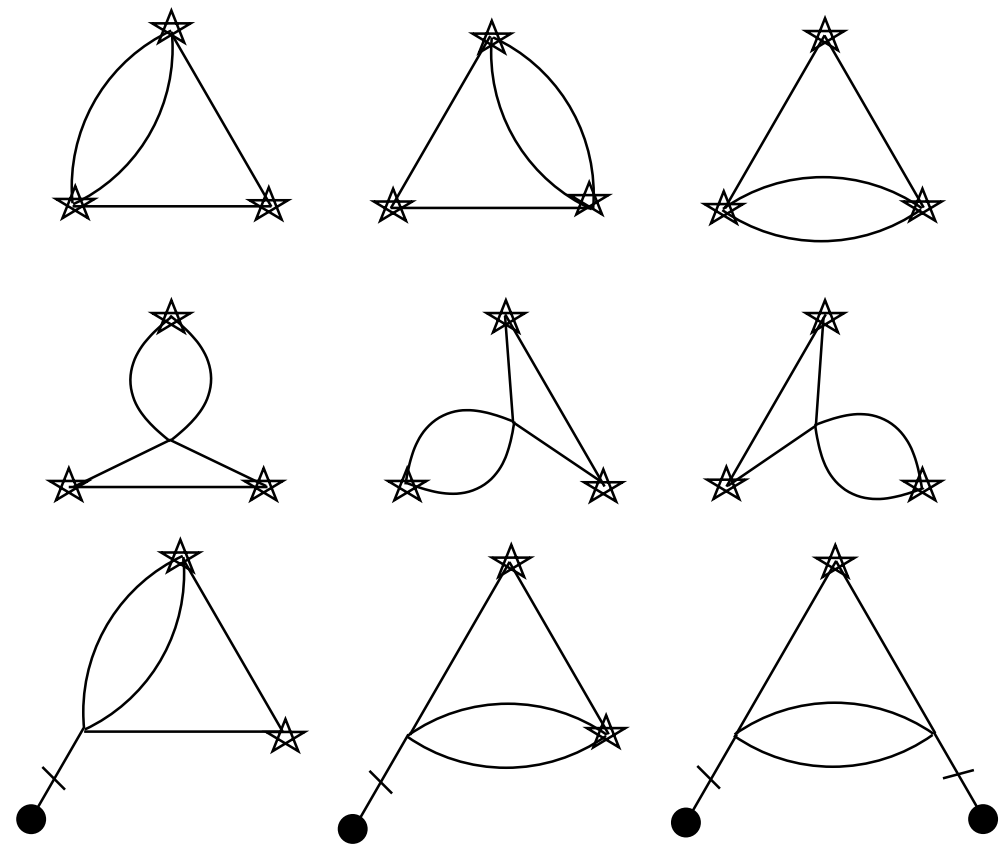

Figure 4: Two-loop graphs for the three-point functions.

Figure 5. Eqs. (86) and (94) allow to prove the validity of the functional equation (66).

Similar arguments can be used in order to prove eqs. (64) and (65) for the rest of the amplitudes in Figure 4 .

\section{More loops}

Here we discuss the röle of the equations of motion eq. (566) in the proof of the validity of eq. (49). The tree level solutions of eq. (47) can be readily constructed from the Feynman vertices given by $\Gamma^{(0)}$ in eq. (52) by the standard procedure recalled in Section 10 .

The crucial point of our approach is the statement that naïve Feynman rules given by $\Gamma^{(0)}$ in eq. (52) yield amplitudes that are solutions of the functional equations (49) for generic D dimensions. Up to two loops the property of dimensional regularization (54)

$$
\int d^{D} k \frac{1}{\left(k^{2}\right)^{\alpha}}=0, \quad \alpha \in \mathcal{C}
$$

implies the validity of the functional equations (49) at the perturbative level by imposing that all tadpole graphs are zero.

For higher number of loops one needs the equations of motion in (56). We illustrate this fact for the four-loop corrections of the amplitude $\Gamma_{J \phi}$. 


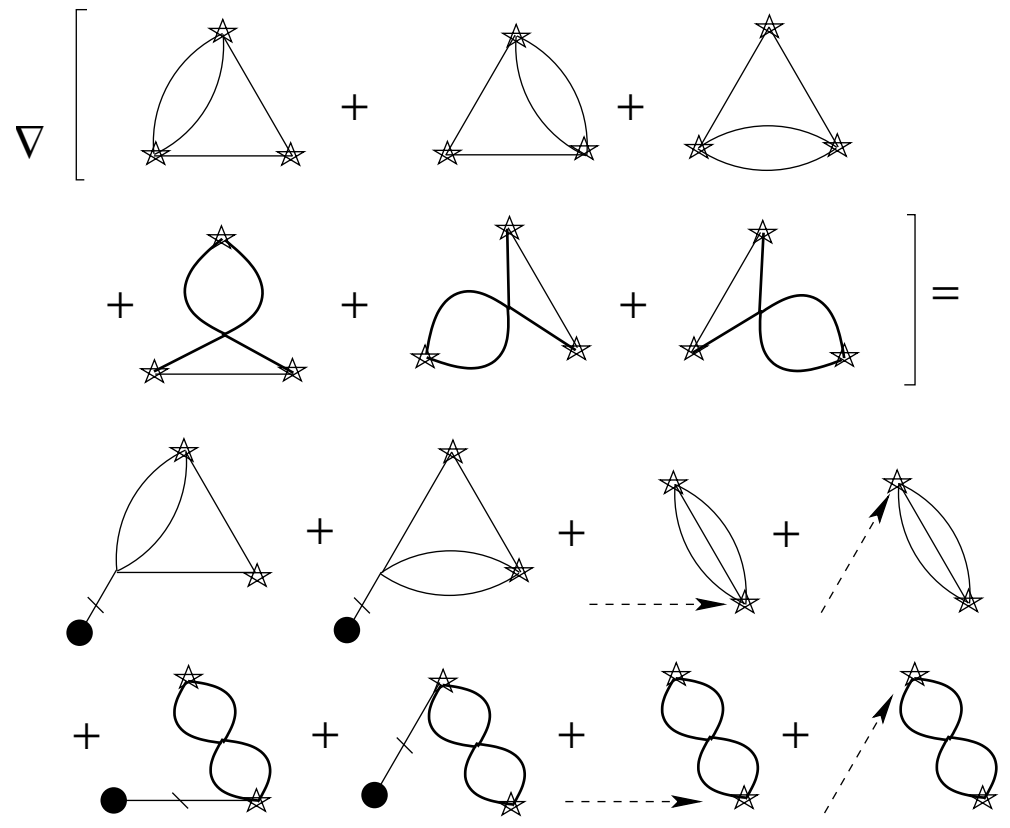

Figure 5: Graphical representation of eq. (66) at two loop level.

The equation to be proved is (62). From the symmetry $\phi_{a} \rightarrow-\phi_{a}$ we see that starting from $F_{a}^{\mu}$ we have three or five $\phi$ lines as depicted in Figures 6 and 17. When one uses the functional identities for these vertices, new terms appear in comparison with the single one present in eq. (62). In particular (94)

$\frac{m_{D}^{2}}{2} \partial^{\mu_{1}} \Gamma_{J_{a_{1}}^{\mu_{1}} \phi_{a_{2}} \phi_{a_{3} \phi_{a_{4}}}^{(0)}}+m_{D} \Gamma_{\phi_{a_{1}} \phi_{a_{2}} \phi_{a_{3}} \phi_{a_{4}}}^{(0)}+\sum_{j=2}^{4} \Gamma_{K_{0} \phi_{a_{j+1}} \phi_{a_{j+2}}}^{(0)} \Gamma_{\phi_{a_{1}} \phi_{a_{j}}}^{(0)}=0$

and

$$
\begin{aligned}
& \frac{m_{D}^{2}}{2} \partial^{\mu_{1}} \Gamma_{J_{a_{1}}^{\mu_{1}} \phi_{a_{2}} \phi_{a_{3}} \phi_{a_{4}} \phi_{a_{5}} \phi_{a_{6}}}^{(0)}+m_{D} \Gamma_{\phi_{a_{1}} \phi_{a_{2}} \phi_{a_{3}} \phi_{a_{4}} \phi_{a_{5}} \phi_{a_{6}}}^{(0)} \\
& +\sum_{\mathcal{P}} \Gamma_{K_{0} \phi_{a_{2}^{\prime}} \phi_{a_{3}^{\prime}}}^{(0)} \Gamma_{\phi_{a_{1}} \phi_{a_{4}^{\prime}} \phi_{a_{5}^{\prime}} \phi_{a_{6}^{\prime}}}^{(0)}+\sum_{j=2}^{6} \Gamma_{K_{0} \phi_{a_{j+1}} \phi_{a_{j+2}} \phi_{a_{j+3}} \phi_{a_{j+4}}}^{(0)} \Gamma_{\phi_{a_{1}} \phi_{a_{j}}}^{(0)}=0
\end{aligned}
$$

where $\left\{a_{2}^{\prime}, a_{3}^{\prime}, a_{4}^{\prime}, a_{5}^{\prime}, a_{6}^{\prime}\right\} \in \mathcal{P}$ is any partition into two sets of 2 and 3 elements.

The last element in eq. (137) gives zero by using eq. (135), while the rest of the unwanted terms cancel by using the equations of motion at the tree level, as depicted in Figures 6 and 7 . Our conjecture is that this cancellation 


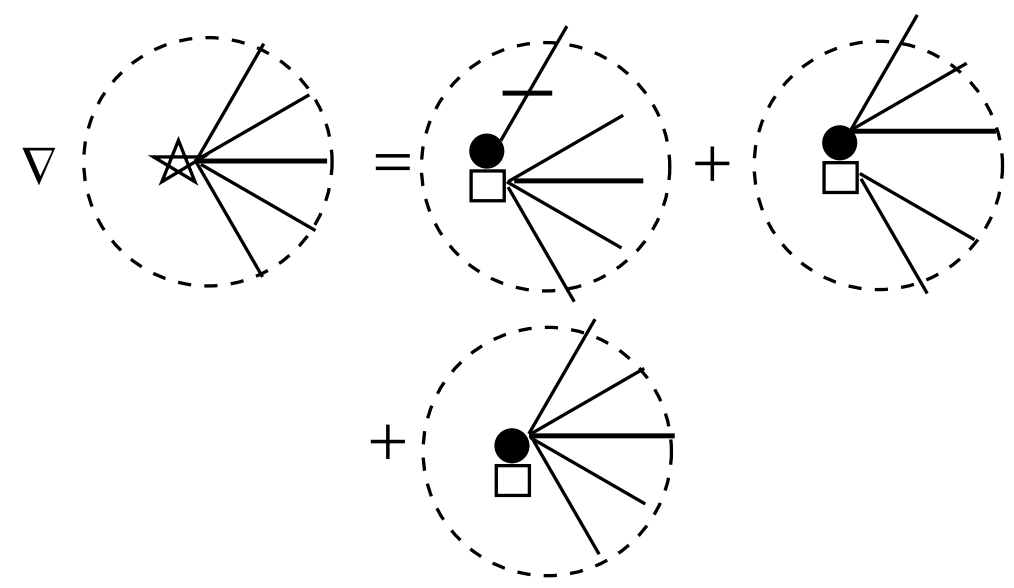

Figure 6: Perturbative representation of the functional identity

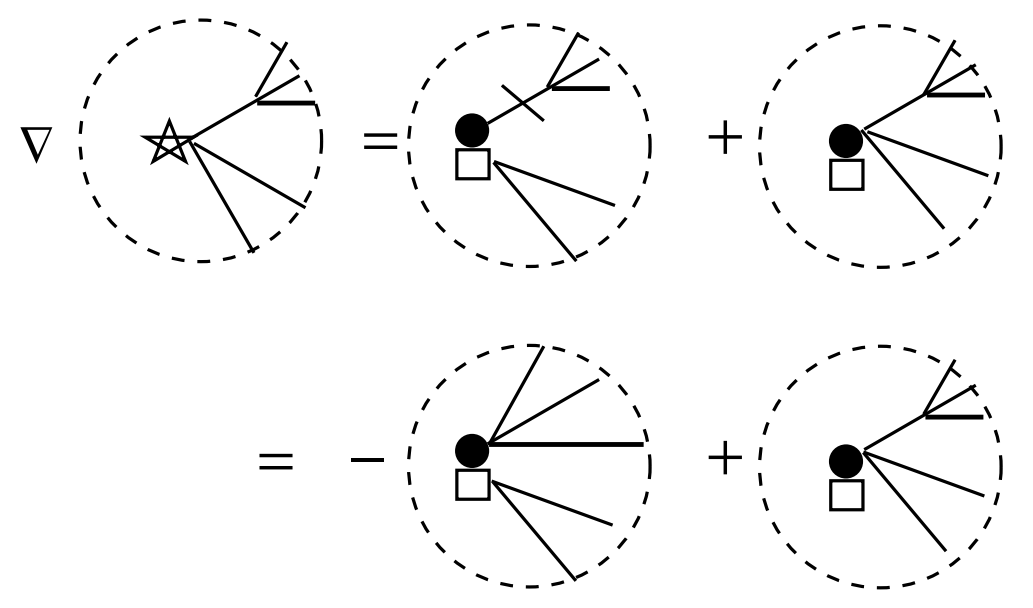

Figure 7: Perturbative check of functional identity

is present at any order in the loop expansion and for any amplitude involving $F_{a}^{\mu}, \phi_{a}$ and $\phi_{0}$.

\section{Subtraction procedure at $D=4$}

In this Section we provide some examples of the subtraction procedure in order to define the amplitudes at $D=4$. For the one loop corrections this amounts to select the pole part, to construct the counterterm by using the normalized amplitudes as in eq. (50) and to get the finite part. In the example involving two loop corrections it will be shown that the recursive subtraction works correctly since the counterterms involve only local terms in the vertex functional. 


\subsection{One loop counterterms for $\Gamma_{J J}, \Gamma_{K_{0} K_{0}}$}

From eq. (104) we see that the pole part of the two-point function involving only the flat connection is

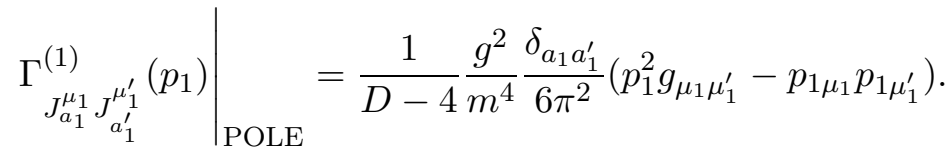

The counterterm has to be evaluated on the normalized amplitude as in eq. (50):

$$
\widehat{\Gamma}^{(1)}[J J]=\frac{1}{(D-4)}\left(\frac{m}{m_{D}}\right)^{2} \frac{g^{2}}{12 \pi^{2} m^{4}} \int d^{D} x J_{a}^{\mu}\left(\square g_{\mu \nu}-\partial_{\mu} \partial_{\nu}\right) J_{a}^{\nu} .
$$

A similar analysis gives the counterterm associated to the amplitude in eq. (106)

$$
\widehat{\Gamma}^{(1)}\left[K_{0} K_{0}\right]=\frac{1}{(D-4)} \frac{3 g^{4}}{2 m^{2}(4 \pi)^{2}} \int d^{D} x K_{0} K_{0} .
$$

14.2 One loop counterterms for $\Gamma_{J J J}, \Gamma_{J J \phi}, \Gamma_{J \phi \phi}, \Gamma_{J \phi K_{0}}, \Gamma_{K_{0} \phi \phi}, \Gamma_{J J K_{0}}$

From eq. (108) the pole part of the three-point flat connection $\Gamma_{J J J}$ is

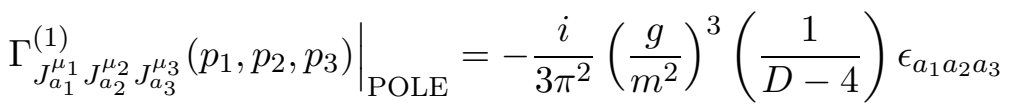

$$
\begin{aligned}
& {\left[\left(p_{3}-p_{2}\right)_{\mu_{1}} g_{\mu_{2} \mu_{3}}+\left(p_{1}-p_{3}\right)_{\mu_{2}} g_{\mu_{1} \mu_{3}}+\left(p_{2}-p_{1}\right)_{\mu_{3}} g_{\mu_{1} \mu_{2}}\right]}
\end{aligned}
$$

Then the counterterm is

$$
\widehat{\Gamma}^{(1)}[J J J]=\frac{1}{3 \pi^{2}}\left(\frac{g}{m^{2}}\right)^{3}\left(\frac{1}{D-4}\right)\left(\frac{m}{m_{D}}\right)^{4} \epsilon_{a b c} \int d^{D} x \partial_{\mu} J_{a \nu} J_{b}^{\mu} J_{c}^{\nu} .
$$

The pole part of the one loop amplitude $\Gamma_{J J \phi}$ can be obtained form eq. (110)

$$
\begin{aligned}
& \Gamma_{\left.\phi_{a_{1}} J_{a_{2}}^{\mu_{2}} J_{a_{3}}^{\mu_{3}}\left(p_{1}, p_{2}, p_{3}\right)\right|_{\mathrm{POLE}}}^{(1)}=-\frac{1}{6 \pi^{2}} \frac{g^{3}}{m^{5}} \frac{1}{D-4} \\
& \epsilon_{a_{1} a_{2} a_{3}}\left[\left(p_{2}^{2} g_{\mu_{2} \mu_{3}}-p_{2 \mu_{2}} p_{2 \mu_{3}}\right)-\left(p_{3}^{2} g_{\mu_{2} \mu_{3}}-p_{3 \mu_{2}} p_{3 \mu_{3}}\right)\right]
\end{aligned}
$$

which corresponds to a counterterm (according to eq. (50))

$$
\begin{aligned}
& \widehat{\Gamma}^{(1)}[J J \phi]=\frac{1}{6 \pi^{2}} \frac{1}{D-4} \frac{g^{3}}{m^{5}}\left(\frac{m}{m_{D}}\right)^{3} \epsilon_{a_{1} a_{2} a_{3}} \\
& \int d^{D} x \phi_{a_{1}} J_{a_{2}}^{\mu_{2}}\left(\square g_{\mu_{2} \mu_{3}}-\partial_{\mu_{2}} \partial_{\mu_{3}}\right) J_{a_{3}}^{\mu_{3}} .
\end{aligned}
$$


The pole part of the one loop amplitude $\Gamma_{J \phi \phi}$ can be obtained form eq. (115). The counterterm according to the rule in eq. (50) is

$$
\widehat{\Gamma}^{(1)}[J \phi \phi]=\frac{1}{D-4} \frac{1}{24 \pi^{2}} \frac{g^{3}}{m^{4}}\left(\frac{m}{m_{D}}\right)^{2} \epsilon_{a b c} \int d^{D} x\left(\square g_{\mu \nu}-\partial_{\mu} \partial_{\nu}\right) J_{c}^{\mu} \quad \phi_{a} \partial^{\nu} \phi_{b} .
$$

The pole part of the one loop amplitude $\Gamma_{J K_{0} \phi}$ can be obtained form eq. (118). The counterterm according to the rule in eq. (50) is

$$
\widehat{\Gamma}^{(1)}\left[J K_{0} \phi\right]=\frac{2}{D-4} \frac{g^{4}}{m^{4}(4 \pi)^{2}}\left(\frac{m}{m_{D}}\right)^{2} \int d^{D} x\left[4 J_{a_{1}}^{\mu} \partial_{\mu} \phi_{a_{1}}+3 \partial_{\mu} J_{a_{1}}^{\mu} \phi_{a_{1}}\right] K_{0} .
$$

The counterterm associated to the amplitude in eq. (121) is

$$
\widehat{\Gamma}^{(1)}\left[K_{0} \phi \phi\right]=\frac{1}{D-4} \frac{g^{4}}{2 m^{3}(4 \pi)^{2}}\left(\frac{m}{m_{D}}\right) \int d^{D} x K_{0}\left(3 \square\left(\phi^{2}\right)-2 \partial^{\mu} \phi_{b} \partial_{\mu} \phi_{b}\right) .
$$

By using the equation (50) and the results already obtained in eqs. (140), (146) and (32) we get

$$
\widehat{\Gamma}^{(1)}\left[J J K_{0}\right]=\frac{1}{D-4} \frac{8 g^{4}}{m^{5}(4 \pi)^{2}}\left(\frac{m}{m_{D}}\right)^{3} \int d^{D} x K_{0} J_{a}^{\mu} J_{a \mu} .
$$

\subsection{A two-loop example: $\Gamma_{J J}^{(2)}$}

The simplest example of two loop amplitude is given by the graphs in Figure 3. The first graph is somewhat trivial since the subgraph counterterms are inserted in a tadpole, i.e. they give no contribution. That is the reason why the amplitudes in eqs. (131) and (133) contain no double poles in $D=4$. In this respect the second graph in Figure 3 is very interesting. In the generic dimension $D$ the amplitude is given by

$$
\begin{aligned}
& \Gamma_{J_{a}^{\mu} J_{b}^{\nu}}^{(2)}=\delta_{a b} \frac{2 g^{4}}{m_{D}^{6}} \frac{-p^{2}}{(D-1)^{2}}\left(-p^{2} g_{\mu \nu}+p_{\mu} p_{\nu}\right) \\
& \frac{1}{\left[-p^{2}\right]^{3-D}} \frac{\Gamma\left(2-\frac{D}{2}\right)^{2}}{(4 \pi)^{D}} \frac{\Gamma\left(\frac{D}{2}-1\right)^{4}}{\Gamma(D-2)^{2}}
\end{aligned}
$$

The proper subgraphs as shown in Figure 3 give the contribution (given by the counterterm in eq. (145))

$$
\left.\Gamma_{J_{a}^{\mu} J_{b}^{\nu}}^{(2)}\right|_{\text {SUBGRAPHS }}=
$$




$$
\begin{aligned}
& =-i \delta_{a b} \frac{2}{D-4} \frac{4}{3(4 \pi)^{2}} \frac{g^{4}}{m_{D}^{4}} \frac{1}{m^{2}} \frac{-p^{2}}{(D-1)}\left(-p^{2} g_{\mu \nu}+p_{\mu} p_{\nu}\right) \\
& \frac{i}{\left[-p^{2}\right]^{2-\frac{D}{2}}} \frac{\Gamma\left(2-\frac{D}{2}\right)}{(4 \pi)^{\frac{D}{2}}} \frac{\Gamma\left(\frac{D}{2}-1\right)^{2}}{\Gamma(D-2)} .
\end{aligned}
$$

The double poles do not cancel. Then one needs a counterterm

$$
\widehat{\Gamma}^{(2)}[J J]=\frac{1}{(D-4)^{2}}\left(\frac{m}{m_{D}}\right)^{2} \int d^{D} x J_{a}^{\mu} \frac{4 g^{4}}{9(4 \pi)^{4} m^{6}} \square\left(\square g_{\mu \nu}-\partial_{\mu} \partial_{\nu}\right) J_{a}^{\nu} .
$$

A straightforward calculation shows that the sum of all the contributions in eqs. (149), (150) and (151) have no double poles. Also the single pole is zero (this guarantees that sugraph counterterms do not induce non local terms). Only the finite part remains (second graph in Figure 3)

$$
\begin{aligned}
& \left.\Gamma_{J_{a}^{\mu} J_{b}^{\nu}}^{(2)}\right|_{\text {RENORM }}=\delta_{a b}\left(-p^{2} g_{\mu \nu}+p_{\mu} p_{\nu}\right)\left[-p^{2}\right] \frac{g^{4}}{m^{6}(4 \pi)^{4}} \\
& \left\{\frac{2}{9} \ln ^{2}\left[\frac{-p^{2}}{m^{2}(4 \pi)}\right]-\frac{4}{3} \ln \left[\frac{-p^{2}}{m^{2}(4 \pi)}\right]\left[-\frac{\gamma}{3}+\frac{2}{9}\right]\right. \\
& \left.+2\left(-\frac{\gamma}{3}+\frac{2}{9}\right)^{2}\right\} .
\end{aligned}
$$

The finite part of the first graph in Figure 3 is

$$
\begin{aligned}
& \left.\Gamma_{J_{a}^{\mu} J_{b}^{\nu}}^{(2)}\right|_{\mathrm{RENORM}}=\delta_{a b} \frac{1}{3}\left(\frac{g^{2}}{m^{3}(4 \pi)^{2}}\right)^{2}\left[-p^{2}\right]\left(-p^{2} g_{\mu \mu^{\prime}}+3 p_{\mu} p_{\mu^{\prime}}\right) \\
& \left\{\ln \left(\frac{\left[-p^{2}\right]}{m^{2}(4 \pi)}\right)+\frac{\gamma}{16}-\frac{29}{128}\right\}+\delta_{a b} \frac{5}{24}\left(\frac{g^{2}}{m^{3}(4 \pi)^{2}}\right)^{2}\left[-p^{2}\right] p_{\mu} p_{\mu^{\prime}}
\end{aligned}
$$

\section{Conclusions and outlook}

We have formulated the nonlinear sigma model in terms of solutions of a functional equation. The functional equation is obtained by constructing a flat connection in terms of the sigma field and then by considering local gauge transformations. The vertex function at the tree level provides the Feynman rules in $\mathrm{D}$ dimensions. We demonstrated that these naïve rules yield amplitudes that satisfy the functional equations in $\mathrm{D}$ dimensions. This fact suggests a simple strategy for the renormalization of the model in $D=4$ consisting in the recursive subtraction of the poles in the Laurent expansion. However some steps of the proof are still at the level of conjecture. In particular one needs a general proof of the statement in Section 13 on the validity of the solution in $\mathrm{D}$ dimensions and also of the same statement after the subtraction procedure. The last point however might follow from the 
quantum action principle as formulated in Reference [25] for dimensional renormalization. 3

With these provisos the final finite theory depends only on two parameters ( $g$ and $m$ ), but the amplitudes are constructed with a particular subtraction procedure that is in principle not unique.

We consider the strategy very promising. It could open a way to attack other non renormalizable theories as non-abelian gauge theories with a Stückelberg mass term or also the nonlinear sigma model with fermions.

\section{Acknowledgments}

We gratefully acknowledge the warm hospitality of the Center for Theoretical Physics at MIT, Boston, where part of this work has been accomplished, and the partial financial support in the frame of a six-year scientific collaboration between MIT and INFN in honor of Bruno Rossi. For stimulating discussions we are very thankful to R. Jackiw, D. Maison, P. Menotti, A. Quadri and A.A. Slavnov.

\section{A One-loop integrals}

Some useful formulas are given in this appendix

$$
\begin{gathered}
I_{2}(p) \equiv \int \frac{d^{D} k}{(2 \pi)^{D}} \frac{1}{k^{2}(p+k)^{2}} \\
=i \int_{0}^{1} d x \frac{1}{(4 \pi)^{\frac{D}{2}}} \frac{\Gamma\left(2-\frac{D}{2}\right)}{\Gamma(2)}\left[-p^{2} x(1-x)\right]^{\left(\frac{D}{2}-2\right)} \\
=\frac{i}{\left[-p^{2}\right]^{2-\frac{D}{2}}} \frac{\Gamma\left(2-\frac{D}{2}\right)}{(4 \pi)^{\frac{D}{2}}} B\left(\frac{D}{2}-1, \frac{D}{2}-1\right) \\
=\frac{i}{\left[-p^{2}\right]^{2-\frac{D}{2}}} \frac{\Gamma\left(2-\frac{D}{2}\right)}{(4 \pi)^{\frac{D}{2}}} \frac{\Gamma\left(\frac{D}{2}-1\right)^{2}}{\Gamma(D-2)} . \\
\int \frac{d^{D} k}{(2 \pi)^{D}} \frac{k_{\mu}}{k^{2}(p+k)^{2}}=-\frac{p_{\mu}}{2} I_{2}(p) . \\
\int \frac{d^{D} k}{(2 \pi)^{D}} \frac{k_{\mu} k_{\nu}}{k^{2}(p+k)^{2}}=\frac{1}{4(D-1)} I_{2}(p)\left(-p^{2} g_{\mu \nu}+D p_{\mu} p_{\nu}\right) . \\
\left\langle 0\left|\left(\phi_{a}(x) \phi_{b}(y)\right)_{+}\right| 0\right\rangle\left\langle 0\left|\left(\phi_{c}(x) \phi_{d}(y)\right)_{+}\right| 0\right\rangle=-\delta_{a b} \delta_{c d} I_{2}(x-y),
\end{gathered}
$$

\footnotetext{
${ }^{3}$ We thank D. Maison for this suggestion.
} 
where

$$
I_{2}(x)=\int \frac{d^{D} p}{(2 \pi)^{D}} e^{-i p x} I_{2}(p),
$$

and the relations

$$
\begin{aligned}
& \left\langle 0\left|\left(\partial_{\nu} \phi_{a}(x) \phi_{b}(y)\right)_{+}\right| 0\right\rangle\left\langle 0\left|\left(\phi_{c}(x) \phi_{d}(y)\right)_{+}\right| 0\right\rangle=-\delta_{a b} \delta_{c d} \frac{1}{2} \partial_{\nu} I_{2}(x-y) \\
& \left\langle 0\left|\left(\partial_{\mu} \partial_{\nu} \phi_{a}(x) \phi_{b}(y)\right)_{+}\right| 0\right\rangle\left\langle 0\left|\left(\phi_{c}(x) \phi_{d}(y)\right)_{+}\right| 0\right\rangle \\
& =\delta_{a b} \delta_{c d} \frac{1}{4(D-1)}\left(\square g_{\mu \nu}-D \partial_{\mu} \partial_{\nu}\right) I_{2}(x-y) \\
& \left\langle 0\left|\left(\partial_{\mu} \phi_{a}(x) \phi_{b}(y)\right)_{+}\right| 0\right\rangle \quad\left\langle 0\left|\left(\partial_{\nu} \phi_{c}(x) \phi_{d}(y)\right)_{+}\right| 0\right\rangle \\
& =-\delta_{a b} \delta_{c d} \frac{1}{4(D-1)}\left(\square g_{\mu \nu}+(D-2) \partial_{\mu} \partial_{\nu}\right) I_{2}(x-y) .
\end{aligned}
$$

The pole part of $I_{2}(x-y)$ is

$$
\left.I_{2}(x-y)\right|_{\mathrm{POLE}}=-i \frac{2}{D-4} \frac{1}{(4 \pi)^{2}} \delta_{D}(x-y) .
$$

More identities

$$
\begin{aligned}
& \left\langle 0\left|\left(\partial_{\mu} \phi_{a}(x) \partial^{\mu} \phi_{b}(y)\right)_{+}\right| 0\right\rangle\left\langle 0\left|\left(\partial_{\nu} \phi_{c}(x) \phi_{d}(y)\right)_{+}\right| 0\right\rangle=0 \\
& \left\langle 0\left|\left(\partial_{\mu} \partial_{\nu} \phi_{a}(x) \phi_{b}(y)\right)_{+}\right| 0\right\rangle\left\langle 0\left|\left(\partial^{\mu} \phi_{c}(x) \phi_{d}(y)\right)_{+}\right| 0\right\rangle \\
& =-\delta_{a b} \delta_{c d} \frac{1}{4} \square \partial_{\nu} I_{2}(x-y)
\end{aligned}
$$

and finally

$$
\begin{aligned}
& \left\langle 0\left|\left(\partial_{\mu} \partial_{\nu} \phi_{a}(x) \phi_{b}(y)\right)_{+}\right| 0\right\rangle\left\langle 0\left|\left(\partial^{\mu} \partial^{\nu} \phi_{c}(x) \phi_{d}(y)\right)_{+}\right| 0\right\rangle \\
& =-\delta_{a b} \delta_{c d} \frac{1}{4} \square^{2} I_{2}(x-y) .
\end{aligned}
$$

\section{B Two-loop integrals}

Now we evaluate the two-loop integrals:

$$
I_{3}\left(p_{1}\right) \equiv \int \frac{d^{D} p_{2}}{(2 \pi)^{D}} \int \frac{d^{D} p_{3}}{(2 \pi)^{D}} \frac{1}{p_{2}^{2} p_{3}^{2}\left(p_{1}+p_{2}+p_{3}\right)^{2}} .
$$

From eq. (154) we get

$$
\begin{aligned}
& I_{3}\left(p_{1}\right)=\frac{i}{(4 \pi)^{\frac{D}{2}}} \frac{\Gamma\left(2-\frac{D}{2}\right)}{\Gamma(2)} B\left(\frac{D}{2}-1, \frac{D}{2}-1\right) \\
& \int \frac{d^{D} p_{2}}{(2 \pi)^{D}} \frac{1}{\left[-\left(p_{1}+p_{2}\right)^{2}\right]^{2-\frac{D}{2}}} \frac{(-1)}{\left(-p_{2}^{2}\right)}
\end{aligned}
$$




$$
\begin{aligned}
& =\frac{-i}{(4 \pi)^{\frac{D}{2}}} \frac{\Gamma\left(2-\frac{D}{2}\right)}{\Gamma(2)} B\left(\frac{D}{2}-1, \frac{D}{2}-1\right) \\
& \int \frac{d^{D} p_{2}}{(2 \pi)^{D}} \int_{0}^{1} d w \frac{w^{1-\frac{D}{2}}}{\left[-\left(p_{2}+w p_{1}\right)^{2}-p_{1}^{2}\left(w-w^{2}\right)\right]^{3-\frac{D}{2}}} B\left(2-\frac{D}{2}, 1\right)^{-1} \\
& =\frac{1}{(4 \pi)^{D}} \frac{\left[\Gamma\left(\frac{D}{2}-1\right)\right]^{3}}{\Gamma\left(\frac{3 D}{2}-3\right)} \frac{\Gamma(3-D)}{\left[-p_{1}^{2}\right]^{3-D}}
\end{aligned}
$$

The only pole in $\mathrm{D}=4$ is in $\Gamma(3-D)$. Now we start from the identity

$$
\left\langle p_{2 \mu}\right\rangle_{3}=\left\langle p_{3 \mu}\right\rangle_{3}
$$

and in the integral over $p_{3}$ we use eq. (155)

$$
\begin{aligned}
& \left\langle p_{2 \mu}\right\rangle_{3}=-\frac{1}{2}\left\langle\left(p_{1 \mu}+p_{2 \mu}\right)\right\rangle_{3} \\
& =-\frac{I_{3}\left(p_{1}\right)}{2} p_{1 \mu}-\frac{1}{2}\left\langle p_{2 \mu}\right\rangle_{3}
\end{aligned}
$$

and then

$$
\left\langle p_{2 \mu}\right\rangle_{3}=-\frac{I_{3}\left(p_{1}\right)}{3} p_{1 \mu} .
$$

In similar fashion we get

$$
\left\langle p_{2 \mu} p_{2 \nu}\right\rangle_{3}=\left\langle p_{3 \mu} p_{3 \nu}\right\rangle_{3}
$$

and in the integral over $p_{3}$ we use eq. (156)

$$
\begin{aligned}
& \left\langle p_{2 \mu} p_{2 \nu}\right\rangle_{3}=\left\langle\frac{1}{4(D-1)}\left(-\left(p_{1}+p_{2}\right)^{2} g_{\mu \nu}+D\left(p_{1}+p_{2}\right)_{\mu}\left(p_{1}+p_{2}\right)_{\nu}\right)\right\rangle_{3} \\
& =\frac{1}{4(D-1)}\left\langle\left(-p_{1}^{2}\left(1-\frac{2}{3}\right) g_{\mu \nu}+D p_{1 \mu} p_{1 \nu}\left(1-\frac{2}{3}\right)+D p_{2 \mu} p_{2 \nu}\right)\right\rangle_{3}
\end{aligned}
$$

i.e.

$$
\left\langle p_{2 \mu} p_{2 \nu}\right\rangle_{3}=\frac{1}{3(3 D-4)}\left(-p_{1}^{2} g_{\mu \nu}+D p_{1 \mu} p_{1 \nu}\right) I_{3}\left(p_{1}\right) .
$$

\section{References}

[1] C. Becchi, A. Rouet and R. Stora, Phys. Lett. B 52 (1974) 344.

G. Curci and R. Ferrari, Nuovo Cim. A 35, 273 (1976).

T. Kugo and I. Ojima, Phys. Lett. B 73, 459 (1978), Progr. Theor. Phys. 60, 1869 (1978).

R. Ferrari and A. Quadri, JHEP 0411, 019 (2004) arXiv:hep-th/0408168.

[2] T. W. B. Kibble, Phys. Rev. 155 (1967) 1554. 
[3] G. 't Hooft, Nucl. Phys. B 35, 167 (1971).

G. 't Hooft and M. J. G. Veltman, Nucl. Phys. B 44, 189 (1972), Nucl. Phys. B 50, 318 (1972).

[4] L. D. Faddeev and V. N. Popov, Phys. Lett. B 25 (1967) 29.

[5] C. Becchi, A. Rouet and R. Stora, see Reference [1] L.V. Tyutin, Lebedev preprint FIAN n.39 (1975).

[6] E. C. G. Stückelberg, Helv. Phys. Helv. Acta 11 (1938), 299.

R. Ferrari, M. Picariello and A. Quadri Phys. Lett. B611, 215 (2005). arXiv:hep-th/0409194

[7] Ketov, Sergei V., "Quantum nonlinear sigma-models", Springer Verlag, Berlin 2000 .

[8] Zinn-Justin, Jean "Quantum field theory and critical phenomena" . - 4. ed. Oxford: Clarendon Press, 2002.

[9] R. E. Cutkosky, J. Math. Phys. 1, 429 (1960).

[10] M. J. G. Veltman, Physica 29, 186 (1963).

[11] J. Gomis and S. Weinberg, Nucl. Phys. B 469, 473 (1996) arXiv:hep-th/9510087.

[12] A.A. Slavnov, "Yang-Mills field with nonzero mass" in proceedings of the conference "Nonlocal, nonlinear and nonrenormalizable field theories". Azau, (1970). Published by JINR (Dubna), 1970, pp. 229-243.

L.D. Faddeev and A.A. Slavnov, Theor. Math. Phys. 8, 843 (1971).

L.D. Faddeev and A.A. Slavnov, Lett. Nuovo Cimento, 8, 117 (1973).

[13] Wolfgang Tomé, Path Integrals on Group Manifold, World Scientific Publishing, Singapore (1998).

[14] A. Salam and J. Strathdee, Phys. Rev. D 2, 2869 (1970).

D. G. Boulware, Ann. Phys. (N.Y.) 56, 140 (1970).

J. M. Charap, Phys. Rev. D 2, 1554 (1970).

J. M. Charap, Phys. Rev. D 3, 1998 (1971).

J. Honerkamp, Nucl. Phys. B 36, 130 (1972).

J. Honerkamp and K. Meetz, Phys. Rev. D 3, 1996 (1971).

I. S. Gerstein, R. Jackiw, S. Weinberg and B. W. Lee, Phys. Rev. D 3, 2486 (1971).

[15] S. Weinberg, Phys. Rev. 166, 1568 (1968).

[16] G. 't Hooft and M. J. G. Veltman, Nucl. Phys. B 44, 189 (1972).

C. G. Bollini and J. J. Giambiagi, Nuovo Cim. B 12, 20 (1972).

G. M. Cicuta and E. Montaldi, Lett. Nuovo Cim. 4, 329 (1972).

[17] E. Brezin, J. Zinn-Justin and J. C. Le Guillou, Phys. Rev. D 14, 2615 (1976). 
[18] G. 't Hooft, "Quantum Gravity And Black Holes," , Cargése 1987, pag 201226. in proceedings in "Nonperturbative quantum field theory"

See also Ref. 8 ]

[19] L. Tătaru, Phys. Rev. D 12, 3351 (1975).

[20] T. Appelquist and C. W. Bernard, Phys. Rev. D 23, 425 (1981).

[21] S.Weinberg, Phys. Rev. Lett. 18, 188 (1967)

S. Weinberg, Phys. Rev. Lett. 18, 507 (1967).

S. Weinberg, Phys. Rev. 166, 1568 (1968).

C. G. . Callan, S. R. Coleman, J. Wess and B. Zumino, Phys. Rev. 177, 2247 (1969).

S. R. Coleman, J. Wess and B. Zumino, Phys. Rev. 177, 2239 (1969).

R. F. Dashen, Phys. Rev. 183, 1245 (1969).

R. F. Dashen and M. Weinstein, Phys. Rev. 183, 1261 (1969).

L. F. Li and H. Pagels, Phys. Rev. Lett. 26, 1204 (1971).

H. Pagels, Phys. Rept. 16, 219 (1975).

J. Gasser and H. Leutwyler, Annals Phys. 158, 142 (1984).

H. Leutwyler, Annals Phys. 235, 165 (1994) arXiv:hep-ph/9311274.

[22] D. Espriu, J. Matias Phys. Lett. B341, 332 (1995)

[23] I.M. Gel'fand and G.E. Shilov, "Generalized functions, Vol. I" , Academic Press, New York, 1966. Ch. I, Sect. 3.8.

D.M. Capper and G. Leibbrandt, J. Math. Phys. 15, 795 (1974); Nuovo Cimento Lett. 6, 117 (1973).

D. M. Capper and G. Leibbrandt, J. Math. Phys. 15, 82 (1974).

G. 't Hooft and M. Veltman, "Diagrammar" CERN Report No. 73-9, 1973.

G. Leibbrandt, Rev. Mod. Phys. 47, 849 (1975).

P. Breitenlohner and D. Maison, Commun. Math. Phys. 52, 39 (1977).

[24] N. N. Bogoliubov and D. V. Shirkov "Introduction to the Theory of Quantized Fields" Wiley, New York (1980).

K. Hepp, Comm. Math. Phys. 2, 301 (1966).

W. Zimmermann, Comm. Math. Phys. 15, 208 (1969).

J. Collins, "Renormalization" Cambridge University Press, (1984).

[25] P. Breitenlohner and D. Maison, Commun. Math. Phys. 52, 11 (1977). 